\title{
A Critical Role for Protein Tyrosine Phosphatase Nonreceptor Type 5 in Determining Individual Susceptibility to Develop Stress-Related Cognitive and Morphological Changes
}

\author{
Chih-Hao Yang, Chiung-Chun Huang, and Kuei-Sen Hsu \\ Department of Pharmacology, College of Medicine, National Cheng Kung University, Tainan 701, Taiwan
}

While stressful life events confer increased risk for the development of psychopathology, most individuals experiencing adversity maintain normal psychological functioning, suggesting that individual differences may influence the susceptibility to develop stress-related psychiatric disorders. However, little is known about what determines this difference between individuals at the molecular level. In the present study, we identify that protein tyrosine phosphatase nonreceptor type 5 (PTPN5) (also known as STEP) is a critical determinant of differences in individual susceptibility to develop stress-related cognitive and morphological changes in rats. Our data demonstrate that ablation of PTPN5 expression delays physiological recovery from stress and augments the development of stress-related cognitive and morphological changes, whereas overexpression of a constitutively active variant of PTPN5 enhances the individual's resilience to stress. Our data also reveal that reduced PTPN5 expression prolongs the duration of extracellular signal-regulated kinase activation, leading to an elevation of $\mathrm{Ca}_{\mathrm{V}} 1.2$ channel expression and a recovery delay of $\mathrm{K}_{\mathrm{V}} 4.2$ channels from inactivation, which in turn heightens neuronal vulnerability to glutamate toxicity. Moreover, intraperitoneal injections of $\mathrm{L}$-type $\mathrm{Ca}^{2+}$ channel blocker nifedipine after stress resulted in a significantly lower rate for developing stress-related cognitive and morphological changes seen in PTPN5 knockdown rats. Together, these results identify a novel role for PTPN5 in mediating the development of stress-related cognitive and morphological changes and suggest that people with PTPN5 deficiency may have a greater susceptibility to capture the deleterious effects of stress.

\section{Introduction}

Stressful life events increase the risk of developing psychopathology such as depression or post-traumatic stress disorder (PTSD). Every individual experiences stressful life events, but individuals show a high degree of heterogeneity of responses (Feder et al., 2009). For some individuals, exposure to acute or chronic stress leads to a pathologically excessive response (also called "allostatic load") and ultimately results in psychopathology. Fortunately, the vast majority of stress-exposed individuals are able to overcome crisis and maintain normal neuropsychological functioning, which is a condition termed "allostasis" (McEwen and Gianaros, 2011). Although why some individuals flourish and others perish under similar stressful conditions remains one of the conundrums in stress research, it is generally believed that promoting successful adaptation of stress is crucial to achieving and maintaining good men-

Received Nov. 27, 2011; revised April 5, 2012; accepted April 9, 2012.

Author contributions: C.-H.Y., C.-C.H., and K.-S.H. designed research; C.-H.Y. and C.-C.H. performed research; C.-H.Y. and C.-C.H. analyzed data; C.-H.Y., C.-C.H., and K.-S.H. wrote the paper.

This work was supported by National Health Research Institute Grant NHRI-EX100-9618NI and National Science Council Grant NSC100-2628-B-006-001-MY4 (Taiwan). C.-C.H. was supported by National Science Council Grant NSC099-2811-B006-023, and C.-H.Y was supported by the National Cheng Kung University "Aim for Top University Project."

Correspondence should be addressed to Dr. Kuei-Sen Hsu, Department of Pharmacology, College of Medicine, National Cheng Kung University, Number 1, University Road, Tainan City 701, Taiwan. E-mail: richard@mail.ncku.edu.tw.

DOI:10.1523/JNEUROSCI.5902-11.2012

Copyright $\odot 2012$ the authors $\quad 0270-6474 / 12 / 327550-13 \$ 15.00 / 0$ tal health. Recently, significant progress has been made in identifying the psychosocial determinants of stress resistance in humans and determining to what extent these factors may be modifiable through clinical interventions (Yehuda et al., 2006). However, little is known about biological factors associated with susceptibility to stress and the molecular mechanisms by which they act in the subsequent development of stress-related disorders.

Protein tyrosine phosphatase nonreceptor type 5 (PTPN5) (also known as STEP) is a brain-enriched protein tyrosine phosphatase preferentially expressed in neurons of the striatum, hippocampus, and cortex (Lombroso et al., 1991; Boulanger et al., 1995). It is a highly conserved member of the PTPN family, with pleiotropic functions in regulating the duration of extracellular signal-regulated kinase (ERK) activation and downstream transcriptional responses after NMDA receptor stimulation (Paul et al., 2003; Valjent et al., 2005), NMDA receptor endocytosis by $\beta$-amyloid (Snyder et al., 2005), and AMPA receptor endocytosis after metabotropic glutamate receptor stimulation (Zhang et al., 2008), suggesting that PTPN5 signaling may function as a tonic suppressor of synaptic strengthening (Pelkey et al., 2002; Tashev et al., 2009). Recently, it has been observed that PTPN5 is severely downregulated in the presence of mutant huntingtin and may participate in the compensatory mechanisms rendering striatal neurons resistant to excitotoxicity (Saavedra et al., 2011). In addition to its role in regulating synaptic strength, our previous study suggested that PTPN5 activity is permissive for novelty 
exploration-induced reversal of stress-induced ERK1/2 hyperphosphorylation and alterations of hippocampal long-term synaptic plasticity (Yang et al., 2006). Given that the harmful effects of acute stress on hippocampal synaptic function are highly correlated with prolonged ERK1/2 activation (Yang et al., 2004, 2006) and activated PTPN5 can dephosphorylate ERK1/2 and therefore limit the ability of ERK1/2 to drive downstream signaling (Paul et al., 2003), we hypothesized that PTPN5 may have a role in determining individual differences in stress responsiveness. Here, we take advantage of a variance in behavioral outcomes after acute stress in outbred Sprague Dawley rats to study the molecular basis of individual differences in the susceptibility to develop stress-related psychiatric disorders. We provide evidence that PTPN5 expression in the dorsal hippocampus (DH) is integrally involved in determining an individual's susceptibility for developing stress-related cognitive and morphological changes following a stress-inducing procedure.

\section{Materials and Methods}

Animals. Male adult Sprague Dawley rats (250-300 g) were used in all experiments. The rats were originally imported from Charles River Laboratories and were bred in our animal facilities. The rats were housed in groups of three in a humidity- and temperature-controlled $\left(25 \pm 1^{\circ} \mathrm{C}\right)$ vivarium on a $12 \mathrm{~h}$ light/dark cycle (lights on 7:00 A.M. to 7:00 P.M.) with access to food and water ad libitum. Rats were acclimated in the animal research facility for at least 1 week before use in behavioral experiments. All behavioral procedures were performed during the light cycle between 10:00 A.M. and 3:00 P.M. All procedures were conducted in accordance with the National Institutes of Health Guide for the Care and Use of Laboratory Animals and were approved by the Institutional Animal Care and Use Committee of National Cheng Kung University.

Stress protocol. Behavioral stress was evoked by 60 tailshocks ( $1 \mathrm{~mA}$ for $1 \mathrm{~s}, 30-90 \mathrm{~s}$ apart) while restrained in a Plexiglas tube (Yang et al., 2004, 2006). This restraint tailshock stress protocol, adapted from the "learned-helplessness" paradigm (i.e., animals are exposed to an unpredictable and uncontrollable aversive stimulus), has been shown extensively to reliably induce behavioral and endocrine signs of stress responses in rats (Kim et al., 1996).

Object location memory test. The experimental apparatus was a black open-field box $\left(40 \times 40 \times 40 \mathrm{~cm}^{3}\right.$, length by width by height), placed in a dimmed illuminated room. During the training trial, the rat was placed in the experimental apparatus and was allowed to explore two identical objects located at two diagonal corners for $10 \mathrm{~min}$. Numerous visual cues including colored star-, moon-, and cross-shaped marks were attached on the walls of the conditioning chamber to provide contextual information. In the test phase, one familiar object was repositioned, and the other familiar object was placed in the same location as during the training trial. The rat was placed in the experimental apparatus for $5 \mathrm{~min}$, and the time spent in exploring each object was recorded using a digital video camera, and scoring was performed with the behavioral tracking system Ethovision (Noldus). To analyze cognitive performance, a discrimination index was calculated for each rat according to the following formula: [time spent on the object in novel location/(time spent on the object in novel location + time spent on the object in familiar location)] $\times 100 \%$.

Sucrose preference test. Rats were first habituated to consume water in the two-bottle choice paradigm for $3 \mathrm{~d}$. At the beginning of the experiment, rats were housed individually in the test chamber identical with their home cage, and water was deprived for $1 \mathrm{~h}$ to increase drinking behavior. In the test session, rats were provided access to two bottles with $1 \%$ sucrose solution and water, respectively, for $2 \mathrm{~h}$. The sucrose preference score was determined by dividing the volume of sucrose consumed by the total liquid consumption.

Open-field test. Rats were placed individually in the center of a test chamber and allowed to freely explore for $10 \mathrm{~min}$ under a low illumination $\left(\sim 10\right.$ lumen $\left./ \mathrm{m}^{2}\right)$. The test chamber consisted of a circular floor $(50$ $\mathrm{cm}$ in diameter) with a $40-\mathrm{cm}$-high wall set on a nonreflective black plastic base. The behavior of the rats was video recorded using a digital video camera and scoring was performed with the behavioral tracking system Ethovision (Noldus). The activity was evaluated based on the number of entries into the central zone, time spent in central zone, and total distance traveled in the open field. After each trial, the apparatus was thoroughly cleaned with $40 \%$ ethanol. The percentage of time spent in the center zone is defined as the percentage of time for the animals exploring the central $25 \%$ ( $25 \mathrm{~cm}$ in diameter) of the chamber.

Resident-intruder test. A resident-intruder paradigm of aggression was used to evaluate the anxiety-like behaviors. In the test session, rats were individually placed in the Plexiglas test chamber $\left(40 \times 20 \times 25 \mathrm{~cm}^{3}\right.$, length by width by height) for $10 \mathrm{~min}$, and then a smaller naive male intruder was placed into the chamber. The offensive aggressive behaviors of the testing rats spent kicking, biting, fight, and clinching in $5 \mathrm{~min}$ with the intruder were recorded to calculate the total duration of attack.

Forced-swimming test. Rats were placed individually in transparent plastic cylinder-shaped bucket ( $30 \mathrm{~cm}$ in diameter and $35 \mathrm{~cm}$ in depth) filled with water at $\sim 25^{\circ} \mathrm{C}$ for a period of $5 \mathrm{~min}$. The behaviors of rats were videorecorded using a digital video camera and scoring was performed with the behavioral tracking system Ethovision (Noldus). An increase in depressive-like behavior was defined as an elevation in time spent immobilization and a decrease in latency to immobility.

Stereotaxic viral injection. Recombinant lentivirus (LV) that expresses PTPN5 or different shRNAs were injected bilaterally into the DH using standard stereotaxic procedures. Under anesthesia with pentobarbital, concentrated virus stock solution was injected into the targeted sites $(0.5$ $\mu \mathrm{l}$ per site at $0.25 \mu \mathrm{l} / \mathrm{min}$ ) by using a Hamilton syringe with a 34 gauge blunted-tip needle. All rats received four sites of viral injection targeting the $\mathrm{CA} 1$ region of the $\mathrm{DH}$ (coordinates: anterioposterior, $-3.8 \mathrm{~mm}$ from bregma; lateral, $\pm 3 \mathrm{~mm}$; ventral, $3 \mathrm{~mm}$; anterioposterior, $-4.5 \mathrm{~mm}$ from bregma; lateral, $\pm 3.5 \mathrm{~mm}$; ventral, $3.2 \mathrm{~mm}$ ) in accordance with the description by Paxinos and Watson (2005). Animals were allowed to recover for $7 \mathrm{~d}$ before starting the experiments. At the end of the experiment, animals used in different behavioral tests were killed by perfusion, and the injection sites were evaluated for each animal. Only those rats that had accurate bilateral injections were chosen for analysis.

Construction and production of engineered LV. High-titer engineered self-inactivating recombinant LV was used for stably gene overexpression or silencing. LV was produced by cotransfection of different lentiviral DNA plasmid with two helper plasmids: vesicular stomatitis virus envelope glycoprotein (VSV-G) and r8.9 in HEK293T cells and medium containing recombinant $\mathrm{LV}$ was harvested $36-48 \mathrm{~h}$ after transfection and ultracentrifuged to get the concentrated lentiviral particles. The pellets were then resuspended by phosphate buffer solution to obtain viral titers of $10^{8} \sim 10^{9} \mathrm{U} / \mathrm{ml}$. For PTPN5 overexpression experiments, fulllength coding region of PTPN5 $\left(\mathrm{STEP}_{46}\right)$ was amplified from a rat brain cDNA library using the primers 5' $5^{\prime}$ ACGCACCGGTCGCATGGAGGAG AAGGTAGAGGA- ${ }^{\prime}$ and $5^{\prime}$-CTAGCAATTGTCACTCTGAGGACTGG AGGG-3', and cloned into a lentiviral expression vector Ubi-X-IRESenhanced green fluorescent protein (EGFP). For PTPN5 knockdown experiments, shRNAs were expressed under a human $\mathrm{H} 1$ promoter and EGFP (as a marker for infection efficacy) under the EF1 $\alpha$ promoter. Three sets of shRNAs targeted at different regions of rat PTPN5 and one control shRNA against DsRed (sh-DsRed) were designed. The following sets of short hairpin sequences were cloned into lentiviral vector using a PCR-based strategy: GCAGGCGGAATTCTTTGAAAT (sh-PTPN5-I), CCTCTGAGTTCCTACATCAAT (sh-PTPN5-II), GATCCAAACATGCG AACAGTA (sh-PTPN5-III), and AGTTCCAGTACGGCTCCAA (shDsRed). To confirm specificity and efficiency of the shRNAs, different sets of lentiviral particles were added into the primary cultures of hippocampal neurons at $5 \mathrm{~d}$ in vitro (DIV) and cell lysates were collected at 15 DIV for Western blot analysis. For the $\mathrm{Ca}_{\mathrm{V}} 1.2$ knockdown experiments, three sets of shRNAs targeting different regions of rat $\mathrm{Ca}_{\mathrm{V}} 1.2$ were designed. The following sets of short hairpin sequences were cloned into lentiviral vector using a PCR-based strategy: CCATTTTCACCATTGAAAT (sh-Ca 1 1.2-I), GGGA CAGTTTGCTCAAGATCC (sh-Ca 1 1.2-II), and CGCCGCAGACAACATC CTC (sh-Ca 1 1.2-III). The specificity and knockdown efficiency of shRNAs were confirmed by Western blot analysis.

Golgi impregnation. Rats were deeply anesthetized with sodium pentobarbital (100 mg/kg, i.p.) and transcardially perfused with PBS, and 
Table 1. List of the primers used for real-time PCR

\begin{tabular}{|c|c|c|c|c|}
\hline Gene name & GenBank accession no. & Forward primer & Reverse primer & Amplicon (bp) \\
\hline Pten & NM_031606 & CCAGGACCAGAGGAAACCT & GCTAGCCTCTGGATTTGA & 240 \\
\hline Dusp1 & NM_053769 & ACCACAAGGCAGACATTAGCTCCT & AAGGTAGTTCAGGGCACTGTTCGT & 389 \\
\hline Ppp1ca & NM_031527 & TTGCCAAGAGGCAGCTGGTG & ACTGCCCATACTTCCCCTTA & 153 \\
\hline Ppp2ca & NM_017039 & TCCGAGTCCCAGGTCAAGAG & GCTACAAGCAGTGTAACTGTTTCA & 236 \\
\hline Ppp3са (PP2B) & NM_017041 & TGCTTCGATTCTCCGACAGG & AAGGCCCACAAATACAGCAC & 204 \\
\hline Ptpn1 (PTP1B) & NM_012637 & GGAAATGGAGAAGGAATTC & TGCTCTTCTGCTCCCACAC & 311 \\
\hline Ptpn5 (STEP) & NM_019253 & TTTCTGGTGGCCTCCGTTA & TCTCAGACTGGGCAGTGGA & 314 \\
\hline Ptpn6 (shp1) & NM_053908 & GGCTAGACTGTGACATTGAC & ATGTTCCCATACTCCGACTC & 187 \\
\hline Ptpn11 (shp2) & NM_013088 & CCCACATCAAGATTCAGAACACT & CTGCCCGTGATGCTCCATGTAA & 107 \\
\hline Ptpra & NM_012763 & AAGAGGCCAGGAGAAGTGTG & GGAGGTCTCGGACAGTGTAG & 119 \\
\hline Ptprb & NM_001108095 & TCGCCTTCTTCATCTGCAGACA & CTTGCAGCTTCATGAAATGC & 180 \\
\hline$C_{v} 1.1$ (Cacna1s) & NM_053873 & GTTACATGAGTTGGATCACACAG & ATGAGCATCTCGATGGTGAAG & 349 \\
\hline$\left(a_{v} 1.2\right.$ (Cacna1c) & NM_012517 & ATGCAAGACGCTATGGGCTAT & CAGGTAGCCTTTGAGATCTTCTTC & 201 \\
\hline$\left(a_{V} 1.3\right.$ (Cacna1d) & NM_017298 & ACATTCTGAACATGGTCTTCACGG & AGGACTTAATGAAGGTCCATAG & 282 \\
\hline$\left(a_{y} 1.4\right.$ (Cacna1f) & NM_053701 & СTCTTCATCTGTGGCAACTACATC & GTACCACCTTCTCCTTGGGTACAA & 307 \\
\hline$\left(a_{v} 2.1\right.$ (Cacna1a) & NM_012918 & GGTCACACCTCACAAGTCCAC & CCAGTCTTCTGGAACATCTCCTG & 306 \\
\hline$\left(a_{v} 2.2(C a c n a 1 b)\right.$ & NM_001195199 & CACCTCGATGAATTCATTCGCGTC & TATCATGAGAGCCGCATAGACCTT & 408 \\
\hline$\left(a_{v} 2.3\right.$ (Cacna1e) & NM_019294 & CGAAAGCATCTCCTCTCTCCGGAT & GTGCAAGGAGTTGGAAGACTCGGT & 381 \\
\hline$\left(a_{v} 3.1\right.$ (Cacna1g) & NM_031601 & GAGCCTGATTTCTTTTCGCC & CGGACATTTCATCTCATGG & 258 \\
\hline$(a, 3.2($ Cacna1h) & NM_153814 & GGTTTGGGTACCATGAACTA & GTAAACTCATAGACTCCGTG & 374 \\
\hline$\left(a_{y} 3.3\right.$ (Cacna1i) & NM_020084 & TATCTTTGGCTGCAAATTCA & TTGGATGAGCTCTGGTCCTC & 306 \\
\hline 18S rRNA & X01117 & CAACTTTCGATGGTAGTCGC & CGCTATTGGAGCTGGAATTAC & 285 \\
\hline
\end{tabular}

then brains were removed and immersed in Golgi-Cox solution at room temperature for 2 weeks. After transferred to a $30 \%$ sucrose solution for $48 \mathrm{~h}, 200-\mu \mathrm{m}$-thick coronal sections were prepared by use of a vibrating microtome (VT1200S; Leica). The slices were subsequently alkalinized in $18.7 \%$ ammonia, fixed in Kodak Rapid Fix solution, dehydrated in alcohol, cleared with xylene, mounted onto gelatinized slides, and coverslipped under Permount (Thermo Fisher Scientific). Spine number was determined blind to the treatment condition. At least 10 dendrites from CA1 pyramidal neurons in each animal were chosen for analysis. The $\mathrm{DH}$ at $3.8-4.6 \mathrm{~mm}$ posterior to bregma was included for the analysis. Images of dendritic spines were taken from the secondary and tertiary branches of basilar dendrites (30-100 $\mu \mathrm{m}$ from the soma) of hippocampal CA1 pyramidal neurons by using the Olympus microscope equipped with a $100 \times, 1.25$ NA oil-immersion objective. Multiple $Z$-stack images of neurons from CA1 regions were collected with the aid of a computer-assisted neuron tracing system (Neurolucida; MicroBrightField) and further reconstructed by using ImageJ software. Spines were defined as dendritic protrusions $\leq 2 \mu \mathrm{m}$ in length, with an obvious head structure. The number of spines were counted with $50 \mu \mathrm{m}$ dendrite segments and presented as the number of dendritic spines in $50 \mu \mathrm{m}$.

Plasma corticosterone assay. Blood samples were obtained from the tail vein just before or at different time points after stress and centrifuged at $1000 \times g$, and plasma was separated and stored at $-20^{\circ} \mathrm{C}$. Plasma corticosterone levels were determined using a commercially available enzyme immunoassay kit (Cayman Chemical) according to the manufacturer's instructions.

Electrophysiological recordings. For the extracellular field potential recordings, hippocampal slices were prepared using standard procedures described previously (Yang et al., 2004, 2006). In brief, rats were killed by decapitation under isoflurane anesthesia, and hippocampal slices (400 $\mu \mathrm{m}$ thick) were prepared using Leica VT1200S vibrating blade microtome (Leica). The slices were placed in a storage chamber of artificial CSF (ACSF) oxygenated with $95 \% \mathrm{O}_{2} / 5 \% \mathrm{CO}_{2}$ and kept at room temperature for at least $1 \mathrm{~h}$ before recording. The composition of the ACSF solution was as follows (in mM): $117 \mathrm{NaCl}, 4.7 \mathrm{KCl}, 2.5 \mathrm{CaCl}_{2}, 1.2 \mathrm{MgCl}_{2}$, $25 \mathrm{NaHCO}_{3}, 1.2 \mathrm{NaH}_{2} \mathrm{PO}_{4}$, and 11 glucose. For recording, one slice was transferred to a submersion-type recording chamber continually perfused with oxygenated ACSF at a flow rate of $2-3 \mathrm{ml} / \mathrm{min}$ at $32.0 \pm 0.5^{\circ} \mathrm{C}$. Extracellular field potential recordings were performed using an Axoclamp-2B amplifier (Molecular Devices). The responses were lowpass filtered at $2 \mathrm{kHz}$, digitally sampled at $5-10 \mathrm{kHz}$, and analyzed using pCLAMP software (version 8.0; Molecular Devices). The evoked post- synaptic responses were induced in CA1 stratum radiatum by stimulation (0.02 ms duration) of Schaffer collateral/commissural afferents at $0.033 \mathrm{~Hz}$ with a bipolar stainless-steel stimulating electrode. Field EPSPs (fEPSPs) were recorded with a glass pipette filled with $1 \mathrm{M} \mathrm{NaCl}(2-3 \mathrm{M} \Omega$ resistance), and the fEPSP slope was measured from $\sim 20-70 \%$ of the rising phase using a least-squares regression. Expression of LTP was induced by high-frequency stimulation, at the test pulse intensity, consisting of two $1 \mathrm{~s}$ trains of stimuli separated by an intertrain interval of $20 \mathrm{~s}$ at $100 \mathrm{~Hz}$. LTD was induced by low-frequency stimulation delivered at $1 \mathrm{~Hz}$ for $15 \mathrm{~min}$ (900 pulses). The magnitudes of LTP and LTD were averaged the responses recorded during the last $10 \mathrm{~min}$ of recording $(50-60 \mathrm{~min})$.

Western blotting. The microdissected hippocampal CA1 tissue samples were lysed in ice-cold Tris- $\mathrm{HCl}$ buffer solution (TBS), pH 7.4, containing a mixture of protein phosphatase and proteinase inhibitors, and ground with a pellet pestle (Kontes Glass). Samples were sonicated and spun down at $15,000 \times g$ at $4^{\circ} \mathrm{C}$ for $10 \mathrm{~min}$. The supernatant was then assayed for total protein concentration using Bio-Rad Bradford Protein Assay Kit. The proteins in each sample were electrophoretically separated in 5 or $10 \%$ SDS-PAGE gel. Following transfer onto nitrocellulose membranes, blots were blocked in TBS containing 3\% bovine serum albumin and $0.1 \%$ Tween 20 for $1 \mathrm{~h}$ and then blotted overnight at $4^{\circ} \mathrm{C}$ with antibodies that recognize PTPN5 (1:1000; Millipore Bioscience Research Reagents), phosphorylated ERK1/2 MAPK (1:1000; Cell Signaling Technology), phosphorylated MEK1/2 (1:2000; Cell Signaling Technology), phosphorylated $\mathrm{K}_{\mathrm{V}} 4.2$ (Thr607; 1:5000; Santa Cruz Biotechnology), $\mathrm{Ca}_{\mathrm{V}} 1.2$ (1:5000; Novus Biologicals), and $\beta$-actin (1:10,000; SigmaAldrich). It was then incubated with HRP-conjugated secondary antibody for $1 \mathrm{~h}$ and developed using the ECL immunoblotting detection system. The immunoblots using phosphorylation site-specific antibodies were subsequently stripped and reprobed with the following antibodies: anti-ERK1/2 antibody (1:1000), anti-MEK1/2 antibody (1:2000), which were purchased from Cell Signaling Technology, and anti- $\mathrm{K}_{\mathrm{V}} 4.2$ antibody (1:500), which was obtained from Abcam. Immunoblots were analyzed by densitometry using Bio-Profil BioLight PC software.

Quantitative real-time PCR. Total RNA was isolated from hippocampal CA1 lysates using an RNeasy Mini Kit (QIAGEN) and treated with RNase-free DNase (RQ1; Promega) to remove potential contamination by genomic DNA. Total RNA $(2 \mu \mathrm{g})$ from tissue lysates was reverse transcribed with the SuperScript cDNA synthesis kit (Invitrogen). PCR was performed on the Roche LightCycler instrument (Roche Diagnostics) using the FastStart DNA Master SYBR Green I kit (Roche Applied Science) according to the manufacturer's instructions. All of the primers 

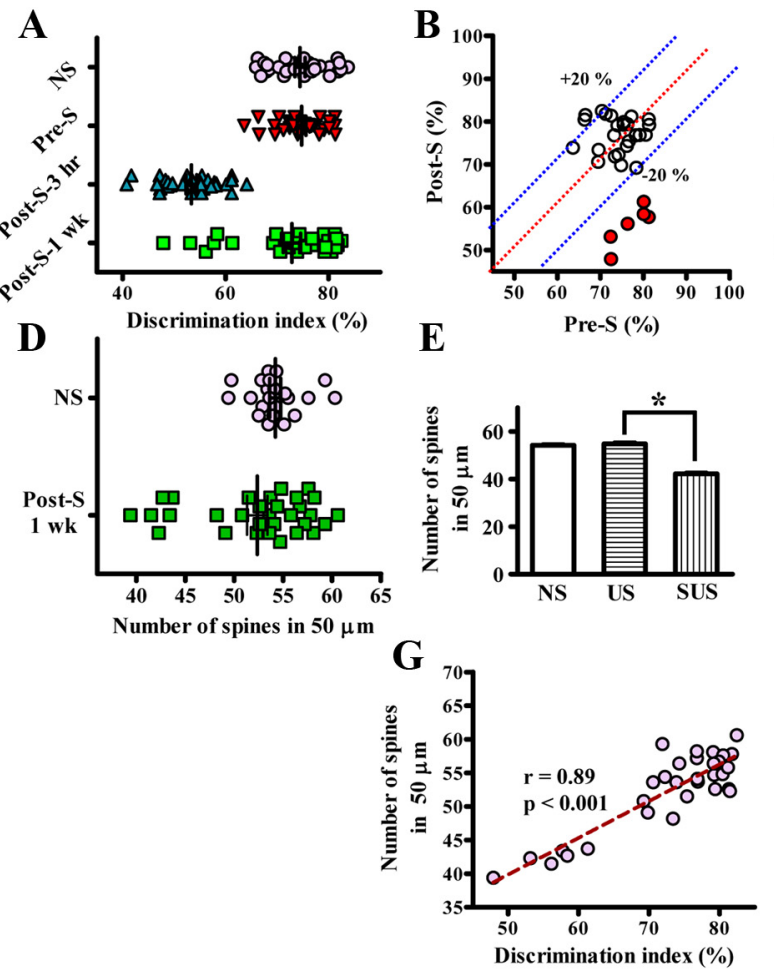

C

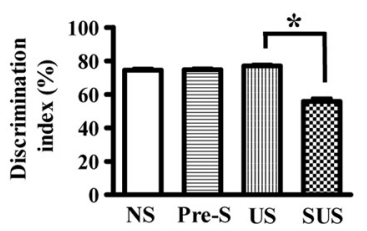

F

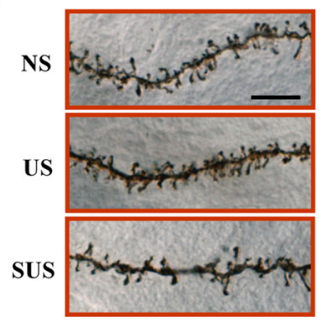

Lactate dehydrogenase release assay. Hippocampal slices $(250 \mu \mathrm{m}$ thick) were prepared with an ice-cold choline-based slicing solution that contained the following (in $\mathrm{mm}$ ): 110 choline chloride, $2.5 \mathrm{KCl}, 1.3 \mathrm{KH}_{2} \mathrm{PO}_{4}, 25 \mathrm{NaHCO}_{3}, 0.5$ $\mathrm{CaCl}_{2}, 7 \mathrm{MgCl}_{2}, 10$ glucose, 1.3 sodium ascorbate, 0.6 sodium pyruvate, and 5.0 kynurenic acid. After their preparation, slices were placed in a holding chamber of ACSF oxygenated with $95 \% \mathrm{O}_{2}$ and $5 \% \mathrm{CO}_{2}$ and kept at room temperature for at least $1 \mathrm{~h}$ before use. The composition of ACSF solution was as follows (in mM): $117 \mathrm{NaCl}$, $4.7 \mathrm{KCl}, 2.5 \mathrm{CaCl}_{2}, 1.2 \mathrm{MgCl}_{2}, 25 \mathrm{NaHCO}_{3}, 1.2$ $\mathrm{NaH}_{2} \mathrm{PO}_{4}$, and 11 glucose at $\mathrm{pH}$ 7.3-7.4. During the experiments, hippocampal slices were first equilibrated in oxygenated ACSF and were then stimulated with different concentrations of glutamate or methyl 2,6-dimethyl-5-nitro-4-[2(trifluoromethyl)phenyl]-1,4-dihydropyridine3-carboxylate (Bay K8644) at $37^{\circ} \mathrm{C}$ for $6 \mathrm{~h}$. After each treatment, the extracellular solutions were collected and lactate dehydrogenase (LDH) activity was quantified using a colorimetric cytotoxicity assay kit (BioVision) according to the manufacturer's instructions. Absorbance data were obtained using a 96-well Molecular Devices Spectramax Microplate Reader (Molecular Devices) with a $450 \mathrm{~nm}$ filter by subtracting $650 \mathrm{~nm}$ as reference wavelength. The maximum LDH release was determined by NMDA ( $50 \mathrm{~mm})$ treatment for $6 \mathrm{~h}$. LDH release in each experimental group was normalized by protein concentration, and the data were presented as percentage relative to the amount of $\mathrm{LDH}$ release caused by NMDA (50 mM).

DNA fragmentation assay. DNA fragmentation assay was performed using a Cell Death Detection ELISA kit (Roche), which is specific for nucleosome-associated cytosolic DNA. Tissue samples were collected after different stimulation, homogenated by lysis buffer, and then centrifuged at $10,000 \times g$ for $15 \mathrm{~min}$ at $4^{\circ} \mathrm{C}$ to obtain the cytoplasmic fraction. Twenty microliter aliquots of the supernatant were added used in the experiments are listed in Table 1. Equal amount of cDNA samples were amplified for 40 cycles. Each cycle consisted of denaturation at $95^{\circ} \mathrm{C}$ for $10 \mathrm{~s}$, annealing at $62^{\circ} \mathrm{C}$ for $10 \mathrm{~s}$, and extension at $72^{\circ} \mathrm{C}$ for $30 \mathrm{~s}$. After amplification, equal volumes of the PCR products were subjected to electrophoresis on $1 \%(\mathrm{w} / \mathrm{v})$ agarose gels and visualized with ethidium bromide. PCR amplifications were assayed in duplicate for each sample. A melting curve was analyzed at the end of the PCR cycle to confirm that a single product had been amplified. Data were analyzed by LightCycler quantification software to determine the threshold cycle above background for each reaction. The relative transcript amount of the gene of interest, which was calculated using standard curves of serial RNA dilutions, was normalized to that of $18 \mathrm{~S}$ rRNA.

PTPN5 activity assay. PTPN5 tyrosine phosphatase activity was assayed by using the commercially available universal tyrosine phosphatase assay ELISA kit (Takara Shuzo) following the manufacturer's instructions. Hippocampal CA1 lysates were prepared as described above but without the addition of protein phosphatase inhibitors. Protein lysates (100 ng) were immunoprecipitated with anti-PTPN5 antibody (1:1000; Millipore Bioscience Research Reagents) at $4^{\circ} \mathrm{C}$ for $3 \mathrm{~h}$ and the resulting immune complex was incubated with a substrate poly(Glu-pTyr) immobilized on microplates in the presence of $5 \mathrm{~mm}$ EDTA-2Na and $50 \mathrm{~mm} \mathrm{NaF}$. After reaction to dephosphorylate of tyrosine residues at $37^{\circ} \mathrm{C}$ for $30 \mathrm{~min}$, the reaction was terminated and the extent of tyrosine phosphorylation was measured by ELISA reader at an optical density of $450 \mathrm{~nm}$ with antiphosphotyrosine (PY20-HRP) and HRP substrate, 3,3',5,5'tetramethylbenzidine.

into a streptavidin-coated 96-well microtiter plate and incubated with 80 $\mu \mathrm{l}$ of antibody mixture for $2 \mathrm{~h}$ at room temperature with mild shaking. The antibody mixture consisted of a mixture of anti-histone biotin and anti-DNA-HRP directed against various histones and antibodies to both single-stranded DNA and double-stranded DNA, which are major constituents of the nucleosomes. After incubation, plates were washed with incubation buffer and the amount of nucleosomes retained by antiDNA-HRP was determined spectrophotometrically with $2,2^{\prime}$-azino-di(3-ethylbenzthiazoline sulfonate)diammonium salt as an HRP substrate. The absorbances of all samples were measured at $405 \mathrm{~nm}$ using a Molecular Devices Spectramax Microplate Reader. Nonspecific signal was determined by subtraction of a reagent blank. The maximum value of cell death was determined by NMDA $(50 \mathrm{~mm})$ treatment for $6 \mathrm{~h}$. The data of cell death in each experimental group were presented as percentage relative to the value of cell death caused by NMDA ( $50 \mathrm{~mm})$.

Pharmacological treatments. 3-(2-Aminoethyl)-5-((4-ethoxyphenyl) methylene)-2,4-thiazolidinedione hydrochloride (ERKI) (SigmaAldrich), an ERK-docking domain inhibitor, was administered $24 \mathrm{~h}$ after stress by intraperitoneal injection to block stress-induced activation of ERK1/2 signaling pathway. Because $5 \mathrm{mg} / \mathrm{kg}$ ERKI was observed effectively to block the ERK1/2-mediated phosphorylation of Elk-1 that occurred after stress, we therefore used this dose for the following behavioral experiments. ERKI was first dissolved in dimethylsulfoxide (DMSO) as stock solution and further diluted in normal saline to $1 \mathrm{mg} / \mathrm{ml}$ for injection. Nifedipine (Tocris Bioscience) was prepared in ethanol (80 


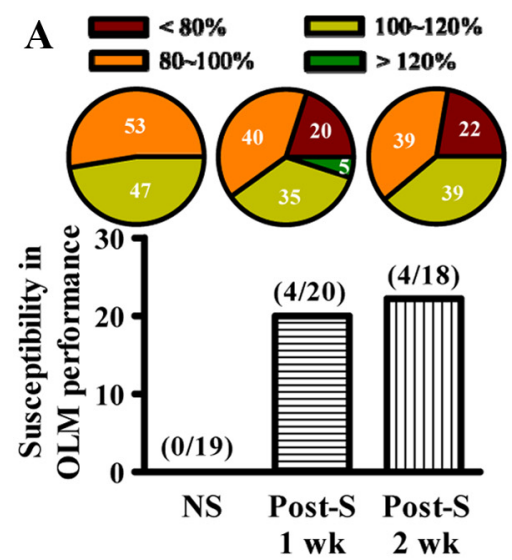

D
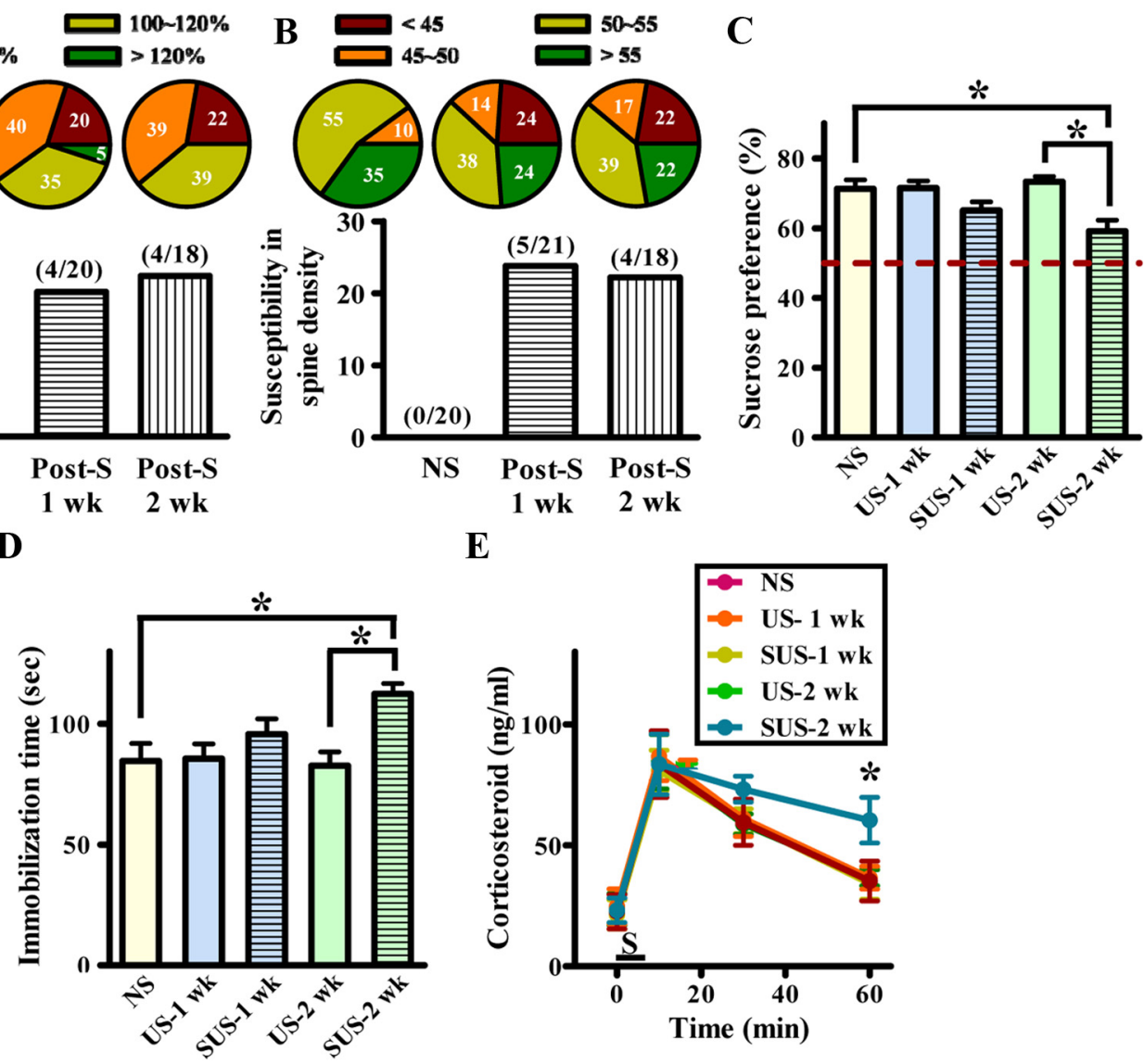

$\mathbf{E}$

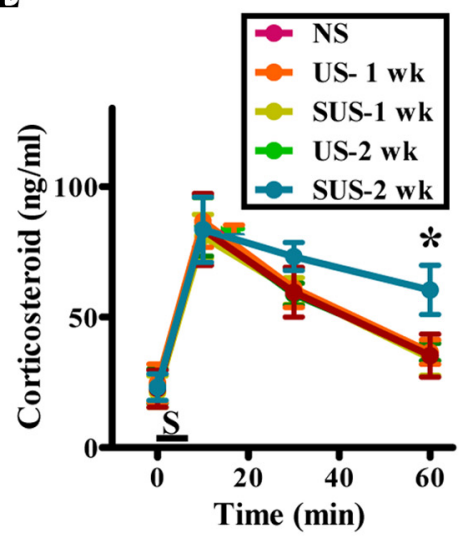

Figure 2. The susceptible group of rats shows depressive-like behaviors and loss of dendritic spines 2 weeks after stress. $A$, Pie chart and bar graph showing the relative distribution of the 0 LM performance in susceptible populations by subtracting poststress values from prestress values at 1 or 2 weeks after stress procedure. The susceptibility was defined as index $<80 \%$. $\boldsymbol{B}$, Pie chart and bar graph showing the relative distribution of dendritic spine density in hippocampal CA1 pyramidal cells of susceptible populations at 1 or 2 weeks after stress procedure. The number $<45$ per 50 $\mu \mathrm{m}$ was classified as susceptible. C, Summary of experiments showing the response in a two-bottle sucrose preference test in US and SUS rats at 1 or 2 weeks after stress procedure ( $n=7$ in each group). $\boldsymbol{D}$, Summary of experiments showing the time spent immobile during the forced-swimming test in US and SUS rats at 1 or 2 weeks after stress procedure ( $n=7$ in each group). $\boldsymbol{E}$, Plasma corticosterone concentration measured immediately before and after 5 min swimming stress ( $n=7$ in each group). The horizontal bar denotes the period of delivery of swimming stress ( 5 min). Each value in parentheses is the number of animals showing susceptibility relative to total number of animals examined. * $p<0.05$. Error bars indicate SEM.

$\mathrm{mg} / \mathrm{ml}$ ) as stock solution and diluted in normal saline to $0.8 \mathrm{mg} / \mathrm{ml}$. Rats were administered intraperitoneally $2 \mathrm{mg} \cdot \mathrm{kg}^{-1} \cdot \mathrm{d}^{-1}$ nifedipine starting 2 or $5 \mathrm{~d}$ after stress. Bay K8644 (Tocris) was prepared as $10 \mathrm{~mm}$ stock solution in DMSO and diluted to final concentrations of 1-10,000 nM.

Statistical analysis. The results are presented as means \pm SEM. The significance of any difference between two groups was calculated using the unpaired Student $t$ test. ANOVA tests were used for multiple-group comparison, and Bonferroni's post hoc analyses were used to assess the significance between isolated groups. The percentage of susceptible rats in the different groups was assessed using a $\chi^{2}$ test. The number of animals used is indicated by $n$. Probability values of $p<0.05$ were considered to represent significant differences.

\section{Results}

Individual differences in susceptibility to stress-related cognitive and morphological changes

Patients with stress-related psychiatric disorders often display persistent cognitive dysfunction that lasts for many weeks or months after the traumatic event (McNally, 2006; Wang et al., 2010). To characterize the molecular basis of susceptibility and resistance to stress-related psychiatric disorders, we adopted an acute, unpredictable, and inescapable restraint tailshock stress paradigm that profoundly impairs hippocampus-dependent spatial tasks such as object location memory (OLM) in rodents (Howland and Cazakoff,
2010; Roozendaal et al., 2010). Male adult Sprague Dawley rats were restrained in a Plexiglas tube and exposed to 60 tailshocks ( $1 \mathrm{~mA}$ for $1 \mathrm{~s}, 30-90 \mathrm{~s}$ apart) and were then tested on their OLM $3 \mathrm{~h}$ and 1 week thereafter. Although all the stressed rats showed OLM impairment at $3 \mathrm{~h}$ after procedure, we observed that only 19\% (6 of 31) of stressed rats still exhibited obvious impairment in OLM at 1 week after the procedure $\left(F_{(2,88)}=0.67 ; p=0.514\right.$; Fig. $\left.1 A\right)$. Because behavioral performances usually show high levels of interindividual variation, we therefore compared the OLM discrimination index before and 1 week after procedure individually. We confirmed that only 19\% (6 of 31) of stressed rats showed markedly impaired OLM performance (Fig. $1 B$ ). Because the vast majority of stressed rats displayed OLM impairment by $20 \%$ at $3 \mathrm{~h}$ after procedure, changes in discrimination index by $>20 \%$ were set as a cutoff: stressed rats showed $\geq 20 \%$ decrease or increase in their OLM performance at 1 week after the procedure compared with their prestress levels were labeled "susceptible" and those with changes in discrimination index $<20 \%$ were labeled "unsusceptible." On the basis of this classification criterion, stressed rats can be segregated into susceptible and unsusceptible populations on the basis of a measure of the recovery from stressinduced impairment of the OLM performance at 1 week after procedure (Fig. 1C). The unsusceptible populations displayed normal OLM performance similar to the nonstressed group. 

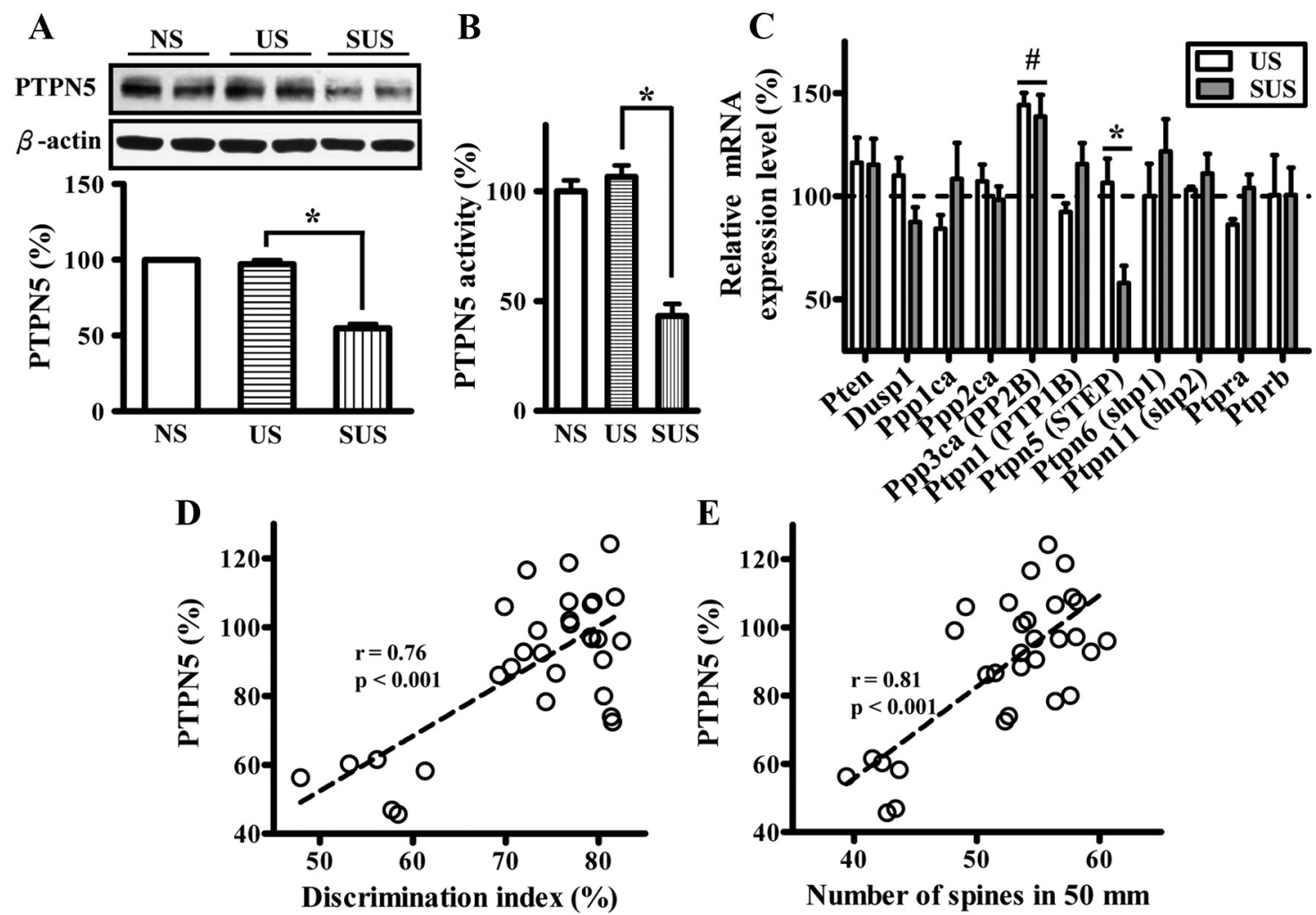

Figure 3. Correlation for the expression of PTPN5 in the hippocampus and the development of stress-related cognitive and morphological changes. $\boldsymbol{A}$, Representative immunoblots and corresponding densitometric analysis showing PTPN5 protein expression in hippocampal CA1 region of NS, US, and SUS rats. B, Summary of experiments showing the PTPN5 activity in hippocampal CA1 tissue lysates from NS, US, and SUS rats ( $n=6-11$ in each group). C, Summary of experiments showing the expression screening for 11 brain-enriched protein tyrosine phosphatases in US and SUS rats at 1 week after stress procedure by quantitative real-time PCR ( $n=4-5$ in each group; ${ }^{*} p<0.05$ compared with control rats; ${ }^{*} p<0.05$ compared with US rats). D, Correlation analysis of the discrimination index in the 0LM and PTPN5 protein expression in the hippocampus at 1 week after stress procedure. $E$, Correlation analysis of the number of dendritic spines in hippocampal CA1 pyramidal cells and PTPN5 protein expression at 1 week after stress procedure. ${ }^{*} p<0.05$. Error bars indicate SEM.

We also analyzed whether the observed stress-induced impairment of the OLM performance in susceptible populations was due to the reduction of their total exploration duration. While a significant decrease in total exploration duration in the OLM tests was observed in susceptible and unsusceptible populations at $3 \mathrm{~h}$ after stress procedure, there were no significant differences between populations $\left(F_{(1,12)}=0.1 ; p=0.83\right)$. When assayed at 1 or 2 weeks after stress procedure, the total exploration durations in the OLM tests of both populations have returned to those measured before exposure to stress (data not shown). Thus, differences in stress-induced impairment of the OLM performance between susceptible and unsusceptible populations cannot be explained by variations in their total exploration duration during the test trials.

The occurrence of stress-related psychiatric disorders has been found to be associated with altered hippocampal structure and function (Yehuda and LeDoux, 2007). Previous functional imaging studies have demonstrated smaller hippocampal volumes in patients with depression and PTSD (Sheline, 2003; Campbell et al., 2004; Bonne et al., 2008; Wang et al., 2010). Hence, we determined whether stress-induced dendritic spine loss might also represent a biological marker for vulnerability to stress. Although the average number of dendritic spines of CA1 pyramidal neurons in stressed rats was comparable with nonstressed rats at 1 week after procedure $(p=0.172)$, we observed that $19 \%$ (6 of 31 ) of stressed rats displayed a significant decrease in dendritic spine density (Fig. 1D), indicating that stressinduced long-lasting decrease in dendritic spine density is also relevant to determine the individual vulnerability to stress (Fig.
$1 E, F)$. Furthermore, there was a significant positive correlation between the OLM performance and the dendritic spine density of CA1 pyramidal neurons in individual rats $(r=0.89$; $p<0.001$; Fig. $1 G)$. Because dendritic spine density on CA1 pyramidal neurons in susceptible populations classified by the OLM performance was $<45$ per $50 \mu \mathrm{m}$, a density of 45 spines $/ 50 \mu \mathrm{m}$ stretch of dendrite was set as a cutoff: stressed rats that showed dendritic spine density $\leq 45$ per $50 \mu \mathrm{m}$ at 1 week after procedure were classified as "susceptible" and those with dendritic spine density $>45$ per $50 \mu \mathrm{m}$ were classified as "unsusceptible." Given that individual differences in susceptibility to develop stress-related psychiatric disorders may be attributable to the differences in their neuroendocrine responses during exposure to stress, we next examined the plasma corticosterone responses to acute stress. However, no significant differences in the neuroendocrine responses to the acute stress were observed between susceptible and unsusceptible rats (data not shown). These findings indicate that measures of the recovery from stressed-induced impairment of the OLM and reduction in the dendritic spines of CA1 pyramidal neurons can be used to segregate susceptible individuals from a general stress-experienced population.

To further assess whether the observed stress-related cognitive and morphological changes in the susceptible group of rats were simply a passive delay of the stress recovery process, we compared the OLM performance and the density of dendritic spines at 1 and 2 weeks after the procedure. However, there were no significant differences between groups in the incidence ratios for stressinduced impairment of OLM performance or the loss of den- 
A

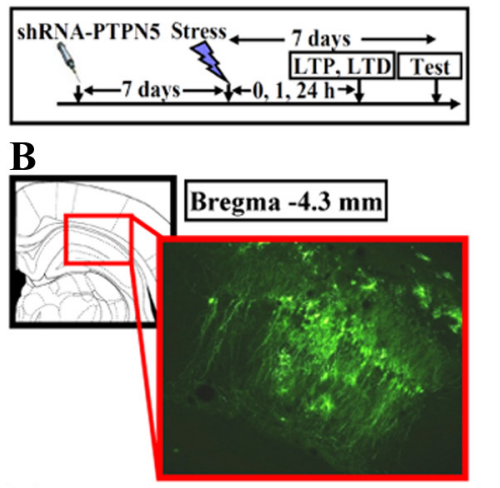

E

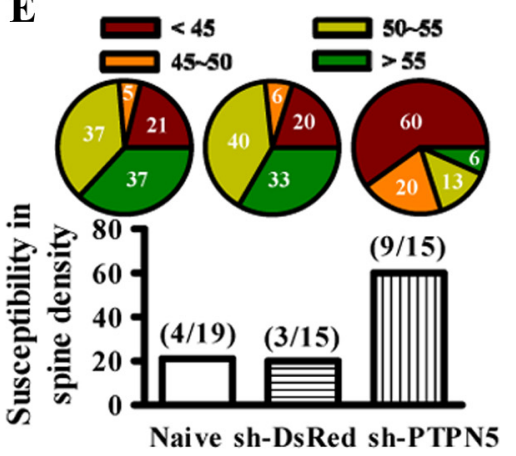

H sh-DsRed $\quad$ sh-PTPN5 I

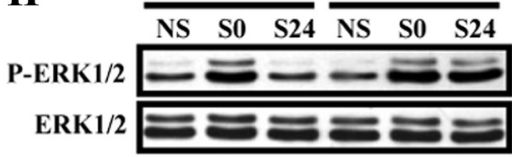

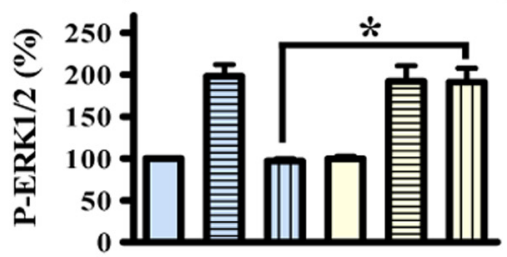

C
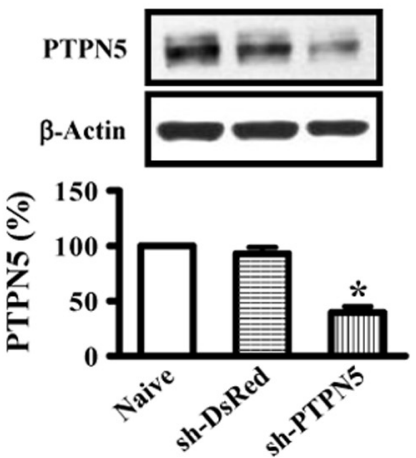

F
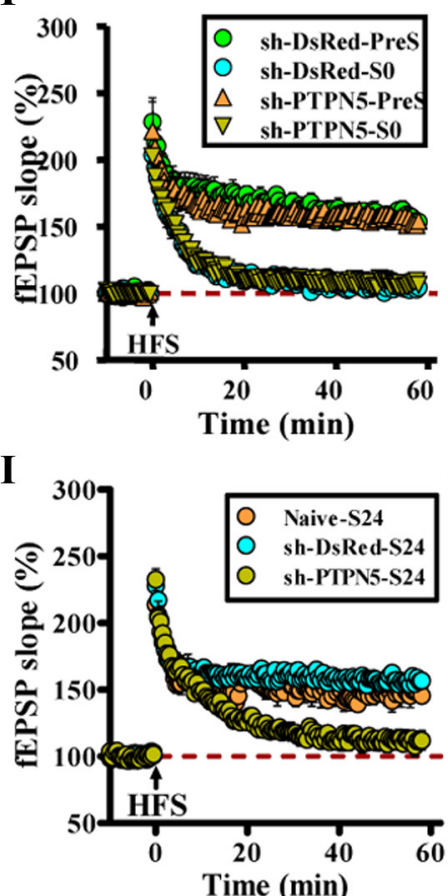

D
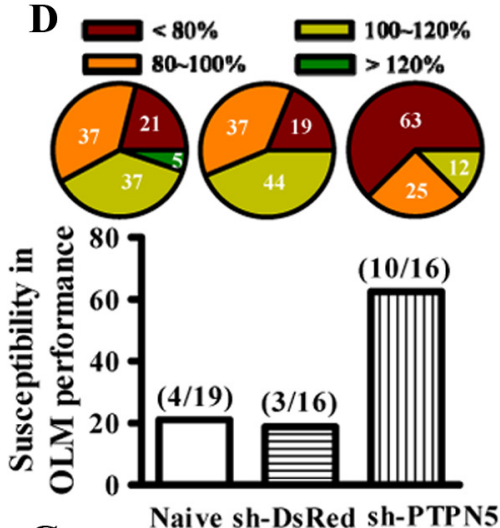

G

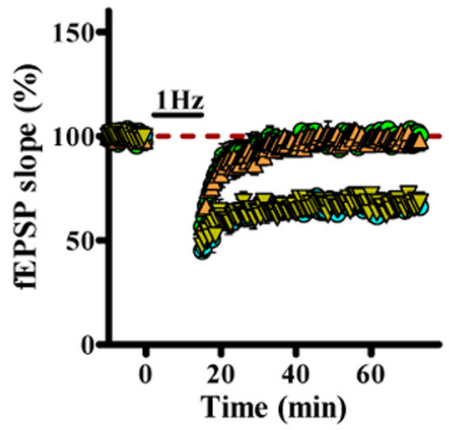

J

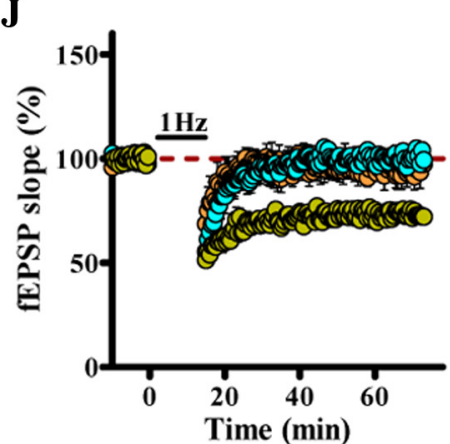

Figure 4. PTPN5 loss of function increases susceptibility to develop stress-related cognitive and morphological changes. $A$, Schematic representation of the experimental designs for examining the stress-induced impairment of long-term synaptic plasticity and the 0 LM in rats receiving bilateral intra-DH injections of sh-DsRed or sh-PTPN 5 before stress treatment. $\boldsymbol{B}$, Representative images showing the delivery of EGFP sh-PTPN5 in the CA1 region of the DH. Scale bar, $500 \mu \mathrm{m}$. C, Representative Western blots confirming stable silencing of PTPN5 expression by sh-PTPN5 $(n=4$ in each group). D, Pie chart and bar graph showing the relative distribution of the OLM performance in rats by subtracting poststress values from prestress values at 1 week after stress procedure. The susceptibility was defined as index $<80 \%$. The naive group represents the individual rat receiving no treatment. $E$, Pie chart and bar graph showing the relative distribution of dendritic spine density in hippocampal CA1 pyramidal cells at 1 week after stress procedure. The number $<45$ per $50 \mu \mathrm{m}$ was classified as susceptible. $\boldsymbol{F}$, Summary of experiments showing the effect of stress on subsequent LTP induction in slices (prepared immediately after stress) from sh-DsRed- or sh-PTPN5-treated rats ( $n=4$ in each group). G, Summary of experiments showing the effect of stress on subsequent LTD induction in slices (prepared immediately after stress) from sh-DsRed- or sh-PTPN5-treated rats ( $n=4$ in each group). $\boldsymbol{H}$, Representative immunoblots and corresponding densitometric analysis showing ERK1/2 phosphorylation in hippocampal CA1 region of sh-DsRed- or sh-PTPN5-treated rats immediately (SO) and $24 \mathrm{~h}$ (S24) after stress ( $n=4)$. I, Summary of experiments showing the induction of LTP in slices from naive, sh-DsRed-treated, or sh-PTPN5-treated rats at $24 \mathrm{~h}$ after stress procedure ( $n=4$ in each group). J, Summary of experiments showing the induction of LTD in slices from naive, sh-DsRed-treated, or sh-PTPN5-treated rats at $24 \mathrm{~h}$ after stress procedure ( $n=4$ in each group). Each value in parentheses is the number of animals showing susceptibility relative to total number of animals examined. ${ }^{*} p<0.05$. Error bars indicate SEM.

dritic spines (Fig. $2 A, B$ ). Interestingly, susceptible rats showed a significant increase in depressive-like behaviors, as indicated by a decrease in sucrose consumption $\left(F_{(4,30)}=6.05 ; p<0.01\right.$; Fig. $2 C)$ and an increase in the duration of immobility during forcedswimming test $\left(F_{(4,30)}=4.24 ; p<0.01 ;\right.$ Fig. $\left.2 D\right)$ as well as poor negative-feedback regulation of hypothalamic-pituitary-adrenal (HPA) axis activity, which was assessed by measuring plasma corticosterone levels at various times before, during, and after exposure to stress (Fig. 2E). Moreover, these behavioral changes may not be derived from the prolonged effects of acute stress, since there were no differences between susceptible and unsus- ceptible rats in aggressive behavior $\left(F_{(4,30)}=0.05 ; p=0.72\right)$ or locomotor activity in the open-field test $\left(F_{(4,30)}=0.1 ; p=0.695\right)$. These results indicate that the observed stress-related cognitive and morphological changes in the susceptible group of rats are not a passive delay of the stress recovery process.

PTPN5 determines individual susceptibility to develop stress-related cognitive and morphological changes PTPN5 counterbalances the activity of ERK1/2 in the hippocampus and has been implicated as a critical regulator of stress responses (Yang et al., 2006). Therefore, we hypothesized that 
A

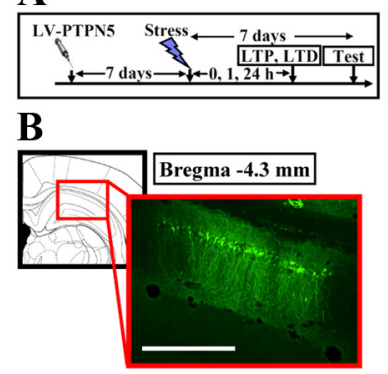

F

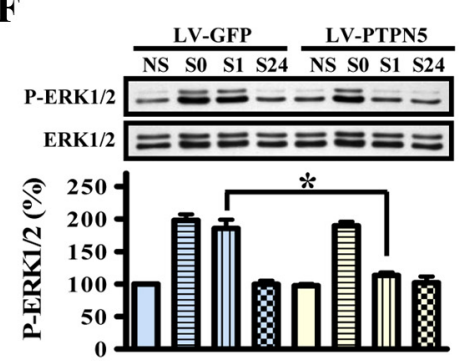

C

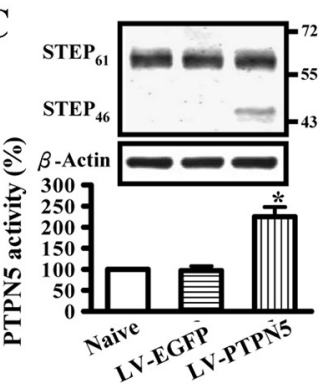

D

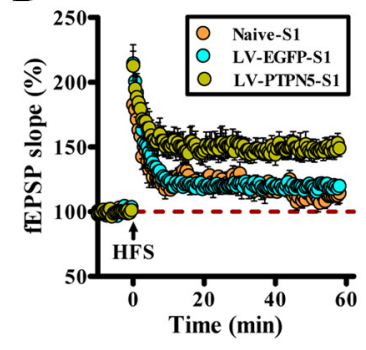

$\mathbf{E}$

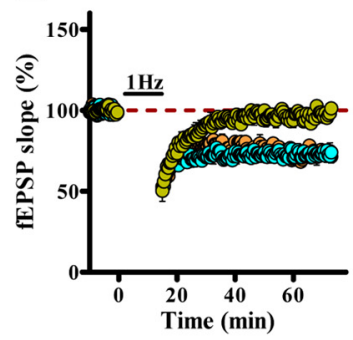

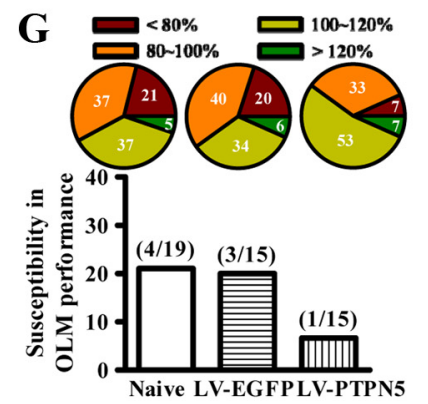

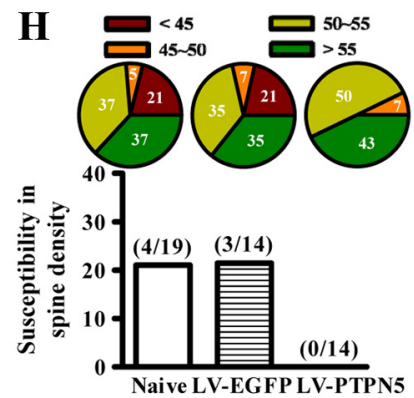

Figure 5. PTPN5 gain of function decreases susceptibility to develop stress-related cognitive and morphological changes. $A$, Schematic representation of the experimental designs for examining the stress-induced impairment of synaptic plasticity and the $0 L M$ in rats receiving bilateral intrahippocampal injections of LV-PTPN 5 before stress treatment. $\boldsymbol{B}$, Representative images showing the delivery of EGFP LV-PTPN5 in the CA1 region of the DH. Scale bar, $500 \mu \mathrm{m}$. C, Representative Western blots and PTPN5 phosphatase activity assay confirming stably overexpressing PTPN5 active variant by LV-PTPN5 ( $n=5$ in each group). $\boldsymbol{D}$, Summary of experiments showing the induction of LTP in slices from naive, LV-EGFP-treated, or LV-PTPN5-treated rats at $1 \mathrm{~h}$ after stress procedure ( $n=4$ in each group). E, Summary of experiments showing the induction of LTD in slices from naive, LV-EGFP-treated, or LV-PTPN5-treated rats at $1 \mathrm{~h}$ after stress procedure ( $n=4$ in each group). $\boldsymbol{F}$, Representative immunoblots and corresponding densitometric analysis showing ERK1/2 phosphorylation in hippocampal CA1 region of LV-EGFP- or LV-PTPN5-treated rats immediately (SO), $1 \mathrm{~h}$ (S1), or $24 \mathrm{~h}($ S24) after stress $(n=4)$. G, Pie chart and bar graph showing the relative distribution of the $0 \mathrm{LM}$ in rats by subtracting poststress values from prestress values at 1 week after stress procedure. The susceptibility was defined as index $<80 \%$. $\boldsymbol{H}$, Pie chart and bar graph showing the relative distribution of dendritic spine density in hippocampal CA1 pyramidal cells at 1 week after stress procedure. The number $<45$ per $50 \mu \mathrm{m}$ was classified as susceptible. Each value in parentheses is the number of animals showing susceptibility relative to total number of animals examined. ${ }^{*} p<0.05$. Error bars indicate SEM.

PTPN5 function in the hippocampus may be a critical determinant of individual susceptibility to stress. Western blot analysis and PTPN5 activity assay showed that the susceptible rats expressed lower levels of PTPN5 protein expression and activity in the DH compared with nonstressed and unsusceptible rats (Fig. $3 A, B$ ). In parallel, we observed that the susceptible rats expressed lower PTPN5 mRNA levels in the DH compared with unsusceptible rats based on quantitative real-time RT-PCR analysis (Fig. 3C). However, no significant differences in the expression levels of other brainenriched protein phosphatases were observed among groups (Fig. $3 C)$. Furthermore, we found that PTPN5 expression levels were significantly correlated with OLM performance $(p<0.01$; Fig. $3 D)$ and the dendritic spine density of CA1 pyramidal neurons $(p<0.01$; Fig. $3 E$ ) in individual rats at 1 week after procedure.

\section{PTPN5 loss of function increases susceptibility to develop} stress-related cognitive and morphological changes

To further validate the association between the low expression of PTPN5 and the expression of susceptible phenotypes, we performed loss-of-function experiments using PTPN5-targeted shRNAs. We injected rats directly into the CA1 region of the $\mathrm{DH}$ with LV coexpressing EGFP and anti-PTPN5 shRNA (shPTPN5) or with control sh-DsRed (Fig. 4A,B). Animals were subjected to the stress paradigm 1 week after the infusion and were then measured using OLM and examined for dendritic spine density of CA1 pyramidal neurons 1 week later. Rats treated with sh-PTPN5 had lower PTPN5 protein levels in hippocampal CA1 tissue lysates than nontreated or sh-DsRed-treated controls (Fig. 4C) but showed normal recall of the OLM and dendritic spine density before stress exposure (data not shown). However, 1 week after the stress procedure, sh-PTPN5-treated rats exhibited significant OLM impairment and a reduction in dendritic spine density. The incidence ratios for stress-induced impairment of OLM performance and the loss of dendritic spine had risen from $19 \%$ (sh-DsRed-treated group) to 63\% (sh-PTPN5-treated group) $(p<$ $0.01 ; \chi^{2}$ test; Fig. $\left.4 D\right)$ and from $20 \%$ (sh-DsRed-treated group) to $60 \%$ (sh-PTPN5-treated group) ( $p<0.01 ; \chi^{2}$ test; Fig. $4 E$ ), respectively. These data strongly implicate PTPN5 in the DH as an important determinant of individual susceptibility for developing stressrelated cognitive and morphological changes.

PTPN5 has been implicated in regulating the duration of ERK1/2 signaling in the hippocampus (Paul et al., 2003; Paul and Connor, 2010). In addition, downstream events linked to ERK1/2 activation have been reported to modulate synaptic transmission and plasticity in the hippocampus (Zhu et al., 2002; Yang et al., 2004). We next examined the contribution of PTPN5 to the occurrence and severity of acute stress reactions by comparing the stress-induced rise in plasma corticosterone levels, ERK1/2 phosphorylation, and alterations of the induction of CA1 LTP and LTD in the DH of sh-PTPN5- and sh-DsRed-treated rats. Notably, there were no significant differences between groups in the levels of acute stress reactions on the induction of CA1 LTP and LTD (Fig. $4 F, G$ ) or ERK1/2 activation (data not shown) when measured immediately after procedure; however, sh-PTPN5- but not sh-DsRed-treated or naive rats still exhibited marked impairment of LTP, enhancement of LTD, and increased ERK1/2 phosphorylation at $24 \mathrm{~h}$ after procedure (Fig. $4 \mathrm{H}-\mathrm{J}$ ), supporting a key role of PTPN5 in turning off stress responses mediated by ERK1/2 signaling pathway. 


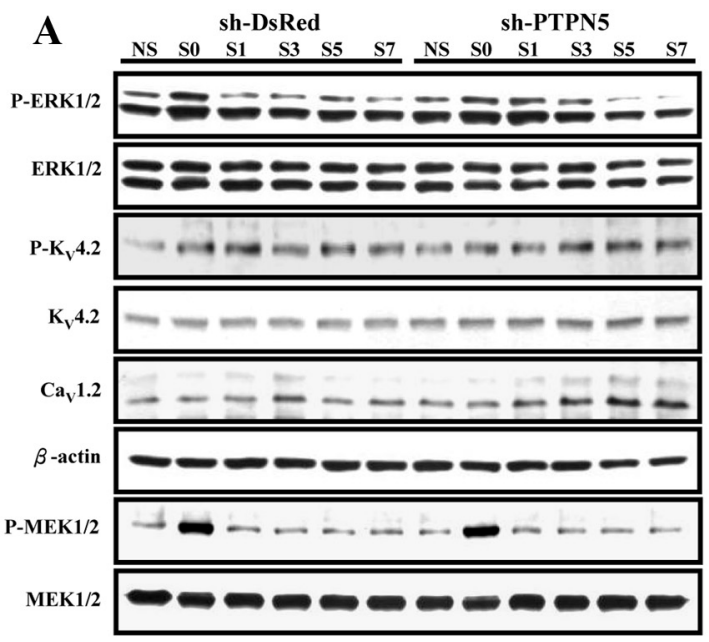

C

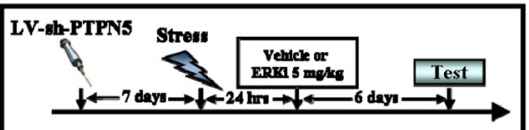

D
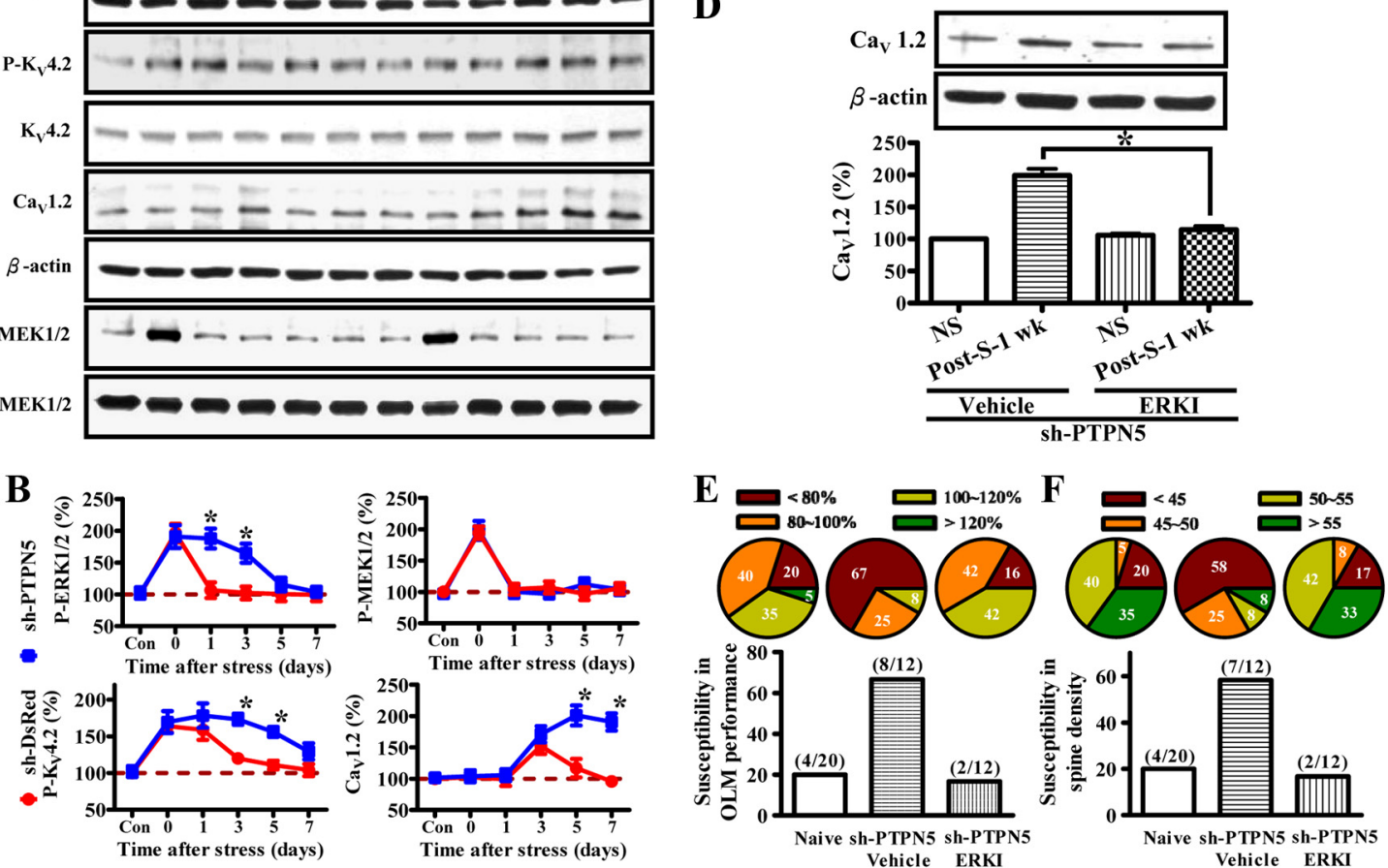

Figure 6. The blockade of ERK1/2 downstream signaling pathway decreases susceptibility to develop stress-related cognitive and morphological changes. $\boldsymbol{A}, \boldsymbol{B}$, Representative immunoblots and corresponding densitometric analysis showing protein phosphorylation or expression levels in hippocampal CA1 region of sh-DsRed- or sh-PTPN5-treated rats at different time points after stress procedure ( $n=5$ ). ${ }^{*} p<0.05$ compared with sh-DsRed group. C, Schematic representation of the experimental designs for examining the stress-induced impairment of the 0LM in rats receiving bilateral intrahippocampal injections of sh-PTPN 5 before stress and ERKI ( $5 \mathrm{mg} / \mathrm{kg}$ ) treatment at $24 \mathrm{~h}$ after stress procedure. $\boldsymbol{D}$, Representative immunoblots and corresponding densitometric analysis showing $\mathrm{Ca}_{\mathrm{y}} 1.2 \mathrm{protein}$ expression in hippocampal CA1 region of sh-PTPN5-treated rats at 1 week after stress procedure with vehicle or ERKI treatment ( $n=4$ in each group). E, Pie chart and bar graph showing the relative distribution of the 0LM in rats by subtracting poststress values from prestress values at 1 week after stress procedure with vehicle or ERKI treatment. The susceptibility was defined as index $<80 \%$. $F$, Pie chart and bar graph showing the relative distribution of dendritic spine density in hippocampal CA1 pyramidal cells at 1 week after stress procedure with vehicle or ERKI treatment. The number $<45$ per $50 \mu \mathrm{m}$ was classified as susceptible. Each value in parentheses is the number of animals showing susceptibility relative to total number of animals examined. Error bars indicate SEM.

PTPN5 gain of function decreases susceptibility to develop stress-related cognitive and morphological changes

We next tested whether genetic enhancement of PTPN5 activity could increase in individual's ability to recover from acute stressing status and reduce in stress-susceptible phenotypes. We virally overexpressed a cytosolic variant of PTPN5 $\left(\mathrm{STEP}_{46}\right.$, sixfold more active than endogenous $\mathrm{STEP}_{61}$ ) (Bult et al., 1996) in the DH (Fig. 5A,B) and then examined stress responsiveness. Because exceeding PTPN5 activity might be accompanied by a concomitant decrease in surface expression of NMDA receptors (Snyder et al., 2005), the viral titers were controlled to yield approximately twofold to threefold increases in PTPN5 activity (Fig. 5C). When LV-EGFP- and LVPTPN5-treated rats were subjected to acute stress, no significant differences between groups were found in the levels of the acute stress response (data not shown). However, rats overexpressing PTPN5 in the DH showed faster recovery from stress compared with naive and LV-EGFP-treated rats (Fig. 5D-F). At $1 \mathrm{~h}$ after the stress procedure, naive and LV-EGFP-treated rats still exhibited LTP impairment, LTD enhancement, and ERK1/2 hyperphosphorylation, whereas LV-PTPN5-treated rats showed normal LTP, LTD, and ERK1/2 phosphorylation. Notably, although overexpressing PTPN5 did not influence the recall of OLM and dendritic spine density before stress exposure (data not shown), we observed a significantly lower rate of the development of stress-related cognitive and morphological changes. The incidence ratios for stress-induced impair- ment of OLM performance and loss of dendritic spine had decreased substantially from 20\% (LV-EGFP-injected group) to $6.7 \%$ (LVPTPN5-treated group) $\left(p<0.01 ; \chi^{2}\right.$ test; Fig. $\left.5 G\right)$ and from $21.4 \%$ (LV-EGFP-treated group) to 0\% (LV-PTPN5-treated group) $(p<$ $0.01 ; \chi^{2}$ test; Fig. $\left.5 H\right)$, respectively.

\section{PTPN5 loss of function increases excitatory overload}

To gain further insight into the molecular events downstream of PTPN5 that contribute to individual susceptibility to develop stress-related psychiatric disorders, we examined the role of ERK1/2-dependent signaling. The sh-PTPN5-treated rats showed significantly prolonged duration of stress-induced ERK1/2 activation, as shown by a long-term increase in ERK1/2 and $\mathrm{K}_{\mathrm{V}} 4.2$ channel phosphorylation at Thr607 (an increase in phosphorylation level indicates suppression of its activity) (Adams et al., 2000; Schrader et al., 2006) compared with sh-DsRedtreated rats (Fig. $6 A, B$ ). In contrast, the MEK1/2 response to stress and its return to baseline were not affected by sh-PTPN5 treatment. Interestingly, we observed a delayed increase in $\mathrm{Ca}_{\mathrm{V}} 1.2$ channel expression after stress challenge, which was significantly promoted in sh-PTPN5-injected rats (Fig. 6A, B). Consistent and significant increases in $\mathrm{Ca}_{\mathrm{V}} 1.2 \mathrm{mRNA}$ were also observed in sh-PTPN5-treated rats after stress (data not shown).

To directly examine the role of ERK-mediated increase of $\mathrm{Ca}_{\mathrm{V}} 1.2$ channel expression and $\mathrm{K}_{\mathrm{V}} 4.2$ channel phosphorylation 
A
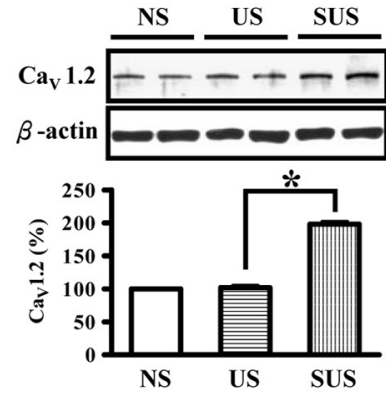

D $\quad$ F

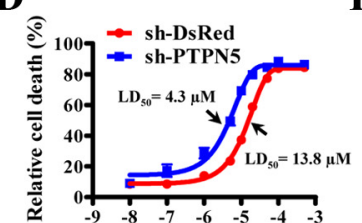

E Glutamate concentration (M) $\mathbf{G}$

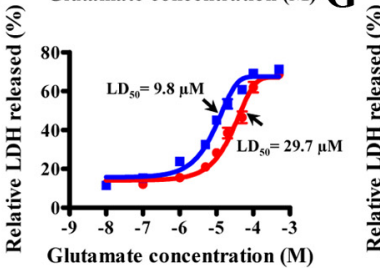

B

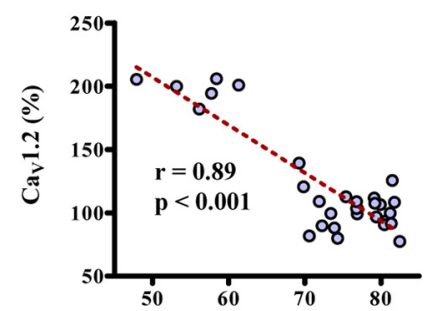

Discrimination index after stress (\%)
C

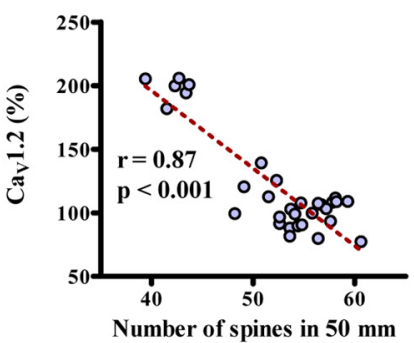

$\mathbf{H}$

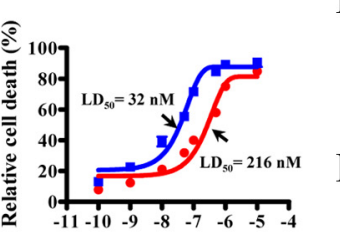

Bay K8644 concentration (M)
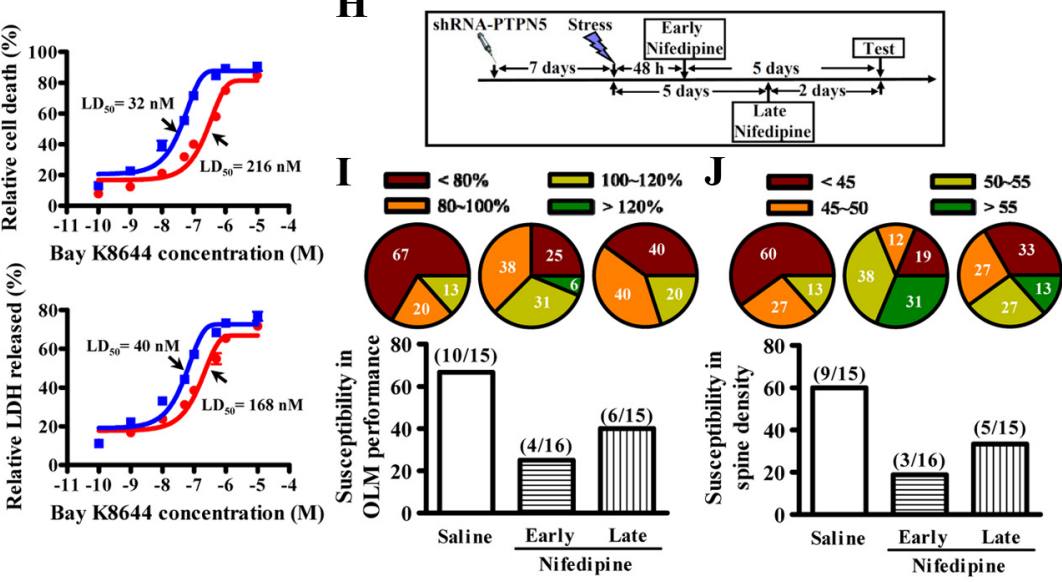

Figure 7. PTPN5 loss of function increases excitatory overload. $A$, Representative immunoblots and corresponding densitometric analysis showing $\mathrm{Ca}_{\mathrm{v}} 1.2$ protein expression in hippocampal $C A 1$ region of NS, US, or SUS rats at 1 week after stress procedure ( $n=4$ in each group). $\boldsymbol{B}$, Correlation analysis of the discrimination index in the OLM and Ca 1.2 protein expression in the DH at 1 week after stress procedure. C, Correlation analysis of the number of dendritic spines in hippocampal CA1 pyramidal cells and Ca 1.2 protein expression in the DH at 1 week after stress procedure. $\boldsymbol{D}, \boldsymbol{E}$, Summary of experiments showing the relative rate of cell death using the DNA fragmentation assay $(\boldsymbol{D})$ or the LDH release assay $(\boldsymbol{E})$ in slices from sh-DsRed-treated or sh-PTPN5-treated rats after treatment with different glutamate concentrations ( $n=5$ in each group). $L_{50}$ represents the median lethal dose. $\boldsymbol{F}, \mathbf{G}$, Summary of experiments showing the relative rate of cell death using the DNA fragmentation assay $(\boldsymbol{F})$ or the LDH release assay $(\boldsymbol{G})$ in slices from sh-DsRed-treated or sh-PTPN5-treated rats after treatment with different Bay K8644 concentrations ( $n=5$ in each group). $\boldsymbol{H}$, Schematic representation of the experimental designs for examining the stress-induced impairment of the OLM in rats receiving bilateral intra-DH injections of sh-PTPN5 before stress and nifedipine $\left(2 \mathrm{mg} \cdot \mathrm{kg}^{-1} \cdot \mathrm{d}^{-1}\right)$ treatment at different time points after stress. $I$, Pie chart and bar graph showing the relative distribution of the 0 LM in rats by subtracting poststress values from prestress values at 1 week after stress procedure with nifedipine or saline treatment. The susceptibility was defined as index $<80 \%$.J, Pie chart and bar graph showing the relative distribution of dendritic spine density in hippocampal CA1 pyramidal cells at 1 week after stress procedure with nifedipine or saline treatment. The number $<45$ per $50 \mu \mathrm{m}$ was classified as susceptible. Each value in parentheses is the number of animals showing susceptibility relative to total number of animals examined. ${ }^{*} p<0.05$. Error bars indicate SEM.

for developing stress-related cognitive and morphological changes in sh-PTPN5-injected rats, we blocked the ERK1/2 downstream signaling pathway in the $\mathrm{DH}$ by administrating ERKI, a novel innovated ERK1/2 docking site inhibitor (Dai et al., 2009; Boston et al., 2011), $24 \mathrm{~h}$ after procedure (Fig. 6C). We found that bilateral infusions of ERKI directly into the DH significantly reduced $\mathrm{Ca}_{\mathrm{V}} 1.2$ channel expression (Fig. $6 \mathrm{D}$ ) and prevented stress-related cognitive and morphological changes in sh-PTPN5-injected rats 1 week after procedure (Fig. 6E,F). The incidence ratios for stress-induced impairment of OLM performance and loss of dendritic spine had decreased substantially from $66.7 \%$ (sh-PTPN5/vehicle group) to $16.7 \%$ (sh-PTPN5/ ERKI group) ( $p<0.01 ; \chi^{2}$ test; Fig. $6 E$ ) and from $58.3 \%$ (shPTPN5/vehicle group) to $16.7 \%$ (sh-PTPN5/ERKI group) ( $p<$ $0.01 ; \chi^{2}$ test; Fig. $6 F$ ), respectively. These results suggest that sustained ERK1/2 activation after stress may contribute, at least in part, to stress-susceptible phenotypes in subjects with lower PTPN5 expression level.

We also confirmed that the susceptible rats showed a significant increase in $\mathrm{Ca}_{\mathrm{V}} 1.2$ channel expression levels compared with nonstressed and unsusceptible rats at 1 week after procedure (Fig. $7 A$ ). The expression levels of $\mathrm{Ca}_{\mathrm{V}} 1.2$ channels were negatively correlated with OLM performance $(p<0.01$; Fig. $7 B)$ and the dendritic spine density of CA1 pyramidal neurons $(p<0.01$; Fig.
$7 C$ ) in individual rat at 1 week after procedure, suggesting that the increase of $\mathrm{Ca}_{\mathrm{V}} 1.2$ through the sustained activation of ERK1/2 signaling may contribute to stress-susceptible phenotypes.

Given that glutamate-induced neuronal death has been implicated as a pathogenic substrate for hippocampal atrophy evident in patients with depression and PTSD (McEwen, 1997; Sapolsky, 2000), we then tested whether increasing $\mathrm{Ca}_{\mathrm{V}} 1.2$ channel expression and $\mathrm{K}_{\mathrm{V}} 4.2$ channel phosphorylation could heighten neuronal vulnerability to glutamate toxicity and lead to development of stress-related cognitive and morphological changes. To test this possibility, fresh hippocampal CA1 tissues from sh-DsRed- or sh-PTPN5-treated rats were collected at $5 \mathrm{~d}$ after procedure, the time point of peak $\mathrm{Ca}_{\mathrm{V}} 1.2$ channel expression and $\mathrm{K}_{\mathrm{V}} 4.2$ channel phosphorylation, and treated with the glutamate or calcium channel agonist Bay K8644 to evaluate the extent of cell death and LDH release. We found that reduced PTPN5 expression by sh-PTPN5 significantly heightened neuronal vulnerability to glutamate and Bay K8644 induced toxicity by shifting the concentration-response curve to the left compared with sh-DsRedtreated rats (Fig. $7 D-G$ ). Moreover, both ERKI and sh-Ca 1.2 treatments effectively reduced the neuronal vulnerability to glutamate toxicity in sh-PTPN5-treated rats (data not shown). Having confirmed an important role for $\mathrm{Ca}_{\mathrm{V}} 1.2$ channels in developing the glutamate toxicity in sh-PTPN5-treated rats, we 
then examined whether pharmacological blockade of L-type $\mathrm{Ca}^{2+}$ channels after stress could prevent the occurrence of stress-susceptible phenotypes. We found that intraperitoneal injections of nifedipine $(2 \mathrm{mg} / \mathrm{kg}) 48 \mathrm{~h}$ (early) or $5 \mathrm{~d}$ (late) after stress resulted in a significantly lower rate for developing stress-related cognitive and morphological changes in sh-PTPN5-treated rats (Fig. $7 \mathrm{H}-\mathrm{J}$ ) compared with a saline-injected group $\left(p<0.01 ; \chi^{2}\right.$ test $)$. Notably, early intervention was more effective than late intervention in preventing stresssusceptible phenotypes.

To further support the role of $\mathrm{Ca}_{\mathrm{V}} 1.2$ in developing stress-related cognitive and morphological changes observed in sh-PTPN5-treated rats, we performed knockdown experiments using $\mathrm{Ca}_{\mathrm{V}} 1.2$ targeted shRNAs $\left(\mathrm{sh}-\mathrm{Ca}_{\mathrm{V}} 1.2\right)$. We injected sh-PTPN5-treated rats directly into the CA1 region of the $\mathrm{DH}$ with sh$\mathrm{Ca}_{\mathrm{V}} 1.2$ or with control sh-DsRed at $24 \mathrm{~h}$ after stress procedure (Fig. 8A). Animals were then measured using OLM and examined for dendritic spine density of CA1 pyramidal neurons $6 \mathrm{~d}$ later. Rats injected with sh-Ca 1.2 had significantly lower $\mathrm{Ca}_{\mathrm{V}} 1.2$ protein levels in hippocampal CA1 tissue lysates than shDsRed-treated controls (Fig. $8 \mathrm{~B}$ ). The incidence ratios for stress-induced impairment of OLM performance and loss of dendritic spine had decreased substantially from $69.2 \%$ (sh-DsRed/sh-PTPN5 group) to $0 \%\left(\mathrm{sh}-\mathrm{Ca}_{\mathrm{V}} 1.2 /\right.$ sh-PTPN5 group) ( $p<0.01 ; \chi^{2}$ test; Fig. $8 C$ ) and from $61.5 \%$ (shDsRed/sh-PTPN5 group) to $7.1 \%$ (sh-Ca $1.2 /$ sh-PTPN5 group) $\left(p<0.01 ; \chi^{2}\right.$ test; Fig. $\left.8 D\right)$, respectively.

\section{Discussion}

The adaptive responses to environmental challenges including both behavioral and neuroendocrine adjustments are critical for maintaining physiological homeostasis, whereas a pathologically excessive response may result in psychopathology (McEwen and Gianaros, 2011). Fortunately, the vast majority of stressexposed individuals are able to overcome crisis and maintain normal neuropsychological functioning. We have shown here for the first time that basal levels of PTPN5 in the DH determine an individual's susceptibility to the development of stress-related cognitive and morphological changes following a stress-inducing procedure. We have also identified two of its downstream substrates, $\mathrm{Ca}_{\mathrm{V}} 1.2$ channels and $\mathrm{K}_{\mathrm{V}} 4.2$ channels, and have implicated them directly in mediating the occurrence of stress-susceptible phenotypes. Our data support a model in which reduced PTPN5 expression may prolong stress-induced ERK1/2 activation, leading to an elevation of $\mathrm{Ca}_{\mathrm{V}} 1.2$ channel expression and a recovery delay of $\mathrm{K}_{\mathrm{V}} 4.2$ channels from inactivation, which in turn heightens neuronal vulnerability to glutamate toxicity and ultimately causes stress-related cognitive and morphological changes.

ERK1/2 is an important component of numerous signaling cascades within neurons. Posttraining activation of ERK1/2 signaling cascades in the hippocampus and amygdala is essential for the con-

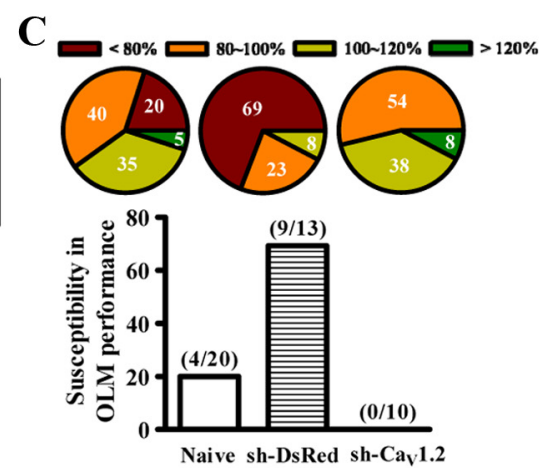

D

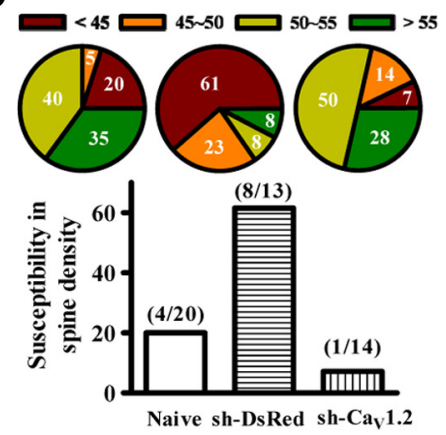

Figure 8. $\quad C a_{v} 1.2$ loss of function decreases stress susceptibility in sh-PTPN5-treated rats. $\boldsymbol{A}$, Schematic representation of the week after stress procedure with sh-DsRed or sh-Ca 1.2 treatment $(n=4$ in each group). C, Pie chart and bar graph showing the distribution of dendritic spine density in hippocampal CA1 pyramidal cells at 1 week after stress procedure with sh-DsRed or sh-Ca 1.2 treatment. The number $<45$ per $50 \mu \mathrm{m}$ was defined as susceptibility. ${ }^{*} p<0.05$. Error bars indicate SEM.

solidation of declarative, spatial, and emotional memories (Sweatt, 2004; Giovannini, 2006). In addition to its role in learning and memory, recent findings from our laboratory have demonstrated a parallel in time course of the increased ERK1/2 activation as well as the effects of acute stress on hippocampal CA1 LTP and LTD; additionally, a pharmacological blockade of ERK1/2 signaling pathway completely prevented the stress effects, strongly suggesting a pivotal role of sustained ERK1/2 activation in mediating the blockade of LTP and the facilitation of LTD induced by the stress (Yang et al., 2004, 2006). It had also been demonstrated that constitutive PTPN5 activity has a significant role in regulating the duration of ERK1/2 activation and its subsequent downstream signaling cascades in preserving the malleability of synapses that have previously been strengthened or depressed (Huang et al., 2005; Yang et al., 2006). The present data extended these findings to demonstrate that knockdown of PTPN5 by RNA interference significantly prolonged the duration of stressinduced ERK1/2 phosphorylation and augmented the development of stress-susceptible phenotypes (Fig. 4), whereas overexpression of PTPN5 promoted the physiological recovery from stress and decreased the susceptibility to develop stress-related cognitive and morphological changes (Fig. 5). It is noteworthy that, although PTPN5 may regulate targets other than ERK1/2, including Fyn phosphorylation (Nguyen et al., 2002) and glutamate receptor trafficking (Snyder et al., 2005), we show that bilateral infusions of ERKI into the DH effectively prevented the development of stresssusceptible phenotypes in sh-PTPN5-treated rats (Fig. 6). These data implicated the regulation by PTPN5 of ERK1/2 activity in the DH as an important mediator of abnormal stress responses following psy- 
chological trauma contributing to the development of stress-related psychiatric disorders.

What could be downstream targets of ERK1/2 in mediating the development of stress-susceptible phenotypes? Since the activated ERK1/2 can promote the activation of many ion channels, neurotransmitter receptors, protein kinases, and transcription factors, the potential candidates are numerous, and it is likely that multiple targets are involved. Although further biochemical investigations will be required to determine the exact targets for ERK1/2, available evidence suggest a strong association between the expression levels of $\mathrm{Ca}_{\mathrm{V}} 1.2$ channels and $\mathrm{K}_{\mathrm{V}} 4.2$ channel phosphorylation in the CA1 region of the $\mathrm{DH}$ and the occurrence of stress-susceptible phenotypes. This view is supported by the following: (1) knockdown of PTPN5 prolonged the duration of stress-induced ERK1/2 phosphorylation along with concomitant increase in $\mathrm{Ca}_{\mathrm{V}} 1.2$ channel expression and $\mathrm{K}_{\mathrm{V}} 4.2$ channel phosphorylation, (2) bilateral $\mathrm{DH}$ infusions of ERKI reduced $\mathrm{Ca}_{\mathrm{V}} 1.2$ channel expression and prevented stress-susceptible phenotypes in sh-PTPN5-treated rats, (3) genetic reduction of $\mathrm{Ca}_{\mathrm{v}} 1.2$ channel expression after stress prevented stressrelated cognitive and morphological changes in sh-PTPN5-treated rats, and (4) pharmacological blockade of L-type $\mathrm{Ca}^{2+}$ channels with nifedipine after stress prevented stress-related cognitive and morphological changes in sh-PTPN5-treated rats. Our results also demonstrate that reduced PTPN5 expression heightened neuronal vulnerability to glutamate and Bay K8644 induced toxicity. This observation is consistent with the view that smaller hippocampal volumes in depression or PTSD patients might result from excessive glutamate-induced excitotoxicity (Golub et al., 2011).

Recent studies have provided evidence that PTPN5 activity can differentially regulate the surface expression of AMPA and NMDA receptors in neurons. In cultured cortical neurons, knockdown of PTPN5 levels by RNA interference has been shown to increase surface expression of NR1, NR2A, and NR2B subunits of NMDA receptors, but not GluR1 subunit of AMPA receptors (Braithwaite et al., 2006). In addition, PTPN5 knock-out mice reveal a significant increase in NR1 and NR2B levels in synaptosomal membrane fractions from the hippocampus (Zhang et al., 2010). PTPN5 has also be found to mediate group I metabotropic glutamate receptor agonist (RS)-3,5-dihydroyphenylglycine-triggered AMPA receptor internalization in both hippocampal slices and primary neuronal cultures (Zhang et al., 2008). Based on these findings, one would predict that knockdown or overexpression of PTPN5 may result in changes in the levels of NMDA receptors or AMPA receptors at synaptic membranes, which in turn alters the ability to induce LTP or LTD. In accordance with this notion, our data indicate that knockdown of PTPN5 by RNA interference produced a slight but significant increase in the levels of GluR1, NR1, and NR2B subunits at synaptic membrane and that overexpression of PTPN5 resulted in a reduction in NR1 and NR2B subunit expression (C.-H. Yang and K.-S. $\mathrm{Hsu}$, unpublished observations). To our surprise, we found no significant differences in the magnitude of LTP or LTD among naive, sh-DsRed-, sh-PTPN5, LV-EGFP-, and LV-PTPN5-treated rats before stress exposure, suggesting that the mild changes in surface expression of glutamate receptors by manipulating PTPN5 were not able to change the induction threshold or expression mechanisms for both LTP and LTD. Zhang et al. (2010) have reported, in contrast to our results, that PTPN5 knock-out mice have enhanced hippocampal CA1 LTP induced by theta-burst stimulation. This discrepancy in findings can be partially reconciled by accounting for differences in the levels of PTPN5 knockdown or protocols for inducing LTP (theta-burst stimulation vs $100 \mathrm{~Hz}$ high-frequency stimulation). We also observed no difference in the expression levels of glutamate receptors at synaptic membrane between susceptible and unsusceptible populations.

These observations beg the question of how could the remodeling of hippocampal dendritic spines contribute to the expression of stress-susceptible phenotypes. The hippocampus has been firmly established in playing a critical role in learning and memory (Jaffard and Meunier, 1993; Knierim et al., 2006), regulating the response of the brain to stress (McEwen, 2002; Sala et al., 2004), and modulating the mesolimbic and mesocortical dopamine reward and motivation circuitries (Lisman and Grace, 2005; Cooper et al., 2006), which all may make contribution to the maintenance of mental health. Because dendritic spines represent the major postsynaptic compartment for excitatory synaptic inputs, changes in the structure and number of dendritic spines may alter the functional activity of hippocampal neurons. A reduction of neuronal activity in the hippocampus might debilitate an individual's ability to process or react to information in new situation and to make decisions about how to deal with new challenges or stressors (McEwen, 2007; McEwen and Gianaros, 2011). Considering that the hippocampus also plays an important role in moderating the HPA axis activity during stress (McEwen, 2002; Sala et al., 2004), an impaired hippocampusdependent negative feedback on the HPA axis may result in elevated HPA axis activity and further exacerbate the actions of adrenal corticosteroids in the long-term effects of repeated and chronic stress exposure (McEwen, 2007; McEwen and Gianaros, 2011). Hence, deterioration of hippocampal neuronal activity may lead not only to the development of cognitive impairments but also to the behavioral sensitization to stress seen in depression or PTSD patients (Yehuda and LeDoux, 2007; Griffin, 2008; McLaughlin et al., 2010; Mahan and Ressler, 2012).

Allostatic systems can promote adaptation to stressful experiences and are most useful when they can be rapidly activated and efficiently terminated afterward. When allostatic responses are no longer terminated appropriately, their long-term effects may have detrimental effects on physical and mental health (McEwen and Gianaros, 2011). Presumably, these short-term (allostasis) and longterm (allostatic load) allodynamic processes can jointly affect vulnerability to develop stress-related psychiatric disorders. In accordance with the concept of allostatic load, we found that individuals who showed susceptibility for developing stress-susceptible phenotypes also exhibited prolonged ERK1/2 activation following stress. Conversely, stress only induced a transient increase in ERK1/2 activation in the unsusceptible group of rats. More importantly, our data indicate that the duration of stress-induced ERK1/2 activation is inversely correlated with PTPN5 protein levels in the DH, suggesting an important role of PTPN5 to shut off allostatic responses mediated by ERK $1 / 2$ signaling pathway after stress.

In summary, our data highlight an important role for PTPN5 in regulating the occurrence of abnormal stress responses underlying depression-related disorders. PTPN5 levels in the DH may serve as a useful biomarker to further our mechanistic understanding of maladaptive stress responses following psychological trauma contributing to the development of stress-susceptible phenotypes. Therapeutic interventions aimed at increasing PTPN5 function or decreasing L-type $\mathrm{Ca}^{2+}$ channel activity may provide beneficial effects in preventing or decreasing the development of stress-related psychiatric disorders.

\section{References}

Adams JP, Anderson AE, Varga AW, Dineley KT, Cook RG, Pfaffinger PJ, Sweatt JD (2000) The A-type potassium channel $\mathrm{K}_{\mathrm{V}} 4.2$ is a substrate for the mitogen-activated protein kinase ERK. J Neurochem 75:2277-2287. Bonne O, Vythilingam M, Inagaki M, Wood S, Neumeister A, Nugent AC, 
Snow J, Luckenbaugh DA, Bain EE, Drevets WC, Charney DS (2008) Reduced posterior hippocampal volume in posttraumatic stress disorder. J Clin Psychiatry 69:1087-1091.

Boston SR, Deshmukh R, Strome S, Priyakumar UD, MacKerell AD Jr, Shapiro P (2011) Characterization of ERK docking domain inhibitors that induce apoptosis by targeting Rsk-1 and caspase-9. BMC Cancer 11:7.

Boulanger LM, Lombroso PJ, Raghunathan A, During MJ, Wahle P, Naegele JR (1995) Cellular and molecular characterization of a brain-enriched protein tyrosine phosphatase. J Neurosci 15:1532-1544.

Braithwaite SP, Adkisson M, Leung J, Nava A, Masterson B, Urfer R, Oksenberg D, Nikolich K (2006) Regulation of NMDA receptor trafficking and function by striatal-enriched tyrosine phosphatase (STEP). Eur J Neurosci 23:2847-2856

Bult A, Zhao F, Dirkx R Jr, Sharma E, Lukacsi E, Solimena M, Naegele JR, Lombroso PJ (1996) STEP61: a member of a family of brain-enriched PTPs is localized to the endoplasmic reticulum. J Neurosci 16:7821-7831.

Campbell S, Marriott M, Nahmias C, MacQueen GM (2004) Lower hippocampal volume in patients suffering from depression: a meta-analysis. Am J Psychiatry 161:598-607.

Cooper DC, Klipec WD, Fowler MA, Ozkan ED (2006) A role for the subiculum in the brain motivation/reward circuitry. Behav Brain Res 174:225-231.

Dai B, Zhao XF, Hagner P, Shapiro P, Mazan-Mamczarz K, Zhao S, Natkunam Y, Gartenhaus RB (2009) Extracellular signal-regulated kinase positively regulates the oncogenic activity of MCT-1 in diffuse large B-cell lymphoma. Cancer Res 69:7835-7843.

Feder A, Nestler EJ, Charney DS (2009) Psychobiology and molecular genetics of resilience. Nat Rev Neurosci 10:446-457.

Giovannini MG (2006) The role of the extracellular signal-regulated kinase pathway in memory encoding. Rev Neurosci 17:619-634.

Golub Y, Kaltwasser SF, Mauch CP, Herrmann L, Schmidt U, Holsboer F, Czisch M, Wotjak CT (2011) Reduced hippocampus volume in the mouse model of posttraumatic stress disorder. J Psychiatr Res 45:650-659.

Griffin MG (2008) A prospective assessment of auditory startle alterations in rape and physical assault survivors. J Trauma Stress 21:91-99.

Howland JG, Cazakoff BN (2010) Effects of acute stress and GluN2Bcontaining NMDA receptor antagonism on object and object-place recognition memory. Neurobiol Learn Mem 93:261-267.

Huang CC, Yang CH, Hsu KS (2005) Do stress and long-term potentiation share the same molecular mechanisms? Mol Neurobiol 32:223-235.

Jaffard R, Meunier M (1993) Role of the hippocampal formation in learning and memory. Hippocampus 3 Spec No:203-217.

Kim JJ, Foy MR, Thompson RF (1996) Behavioral stress modifies hippocampal plasticity through $\mathrm{N}$-methyl-D-aspartate receptor activation. Proc Natl Acad Sci U S A 93:4750-4753.

Knierim JJ, Lee I, Hargreaves EL (2006) Hippocampal place cells: parallel input streams, subregional processing, and implications for episodic memory. Hippocampus 16:755-764.

Lisman JE, Grace AA (2005) The hippocampal-VTA loop: controlling the entry of information into long-term memory. Neuron 46:703-713.

Lombroso PJ, Murdoch G, Lerner M (1991) Molecular characterization of a protein-tyrosine-phosphatase enriched in striatum. Proc Natl Acad Sci U S A 88:7242-7246.

Mahan AL, Ressler KJ (2012) Fear conditioning, synaptic plasticity and the amygdala: implications for posttraumatic stress disorder. Trends Neurosci 35:24-35.

McEwen BS (1997) Possible mechanisms for atrophy of the human hippocampus. Mol Psychiatry 2:255-262.

McEwen BS (2002) Sex, stress and the hippocampus: allostasis, allostatic load and the aging process. Neurobiol Aging 23:921-939.

McEwen BS (2007) Physiology and neurobiology of stress and adaptation: central role of the brain. Physiol Rev 87:873-904.

McEwen BS, Gianaros PJ (2011) Stress- and allostasis-induced brain plasticity. Annu Rev Med 62:431-445.

McLaughlin KA, Conron KJ, Koenen KC, Gilman SE (2010) Childhood adversity, adult stressful life events, and risk of past-year psychiatric disorder: a test of the stress sensitization hypothesis in a population-based sample of adults. Psychol Med 40:1647-1658.

McNally RJ (2006) Cognitive abnormalities in post-traumatic stress disorder. Trends Cogn Sci 10:271-277.

Nguyen TH, Liu J, Lombroso PJ (2002) Striatal enriched phosphatase 61 dephosphorylates Fyn at phosphotyrosine 420. J Biol Chem 277: 24274-24279.
Paul S, Connor JA (2010) NR2B-NMDA receptor-mediated increases in intracellular $\mathrm{Ca}^{2+}$ concentration regulate the tyrosine phosphatase, STEP, and ERK MAP kinase signaling. J Neurochem 114:1107-1118.

Paul S, Nairn AC, Wang P, Lombroso PJ (2003) NMDA-mediated activation of the tyrosine phosphatase STEP regulates the duration of ERK signaling. Nat Neurosci 6:34-42.

Paxinos G, Watson C (2005) The rat brain in stereotaxic coordinates. San Diego: Elsevier Academic.

Pelkey KA, Askalan R, Paul S, Kalia LV, Nguyen TH, Pitcher GM, Salter MW, Lombroso PJ (2002) Tyrosine phosphatase STEP is a tonic brake on induction of long-term potentiation. Neuron 34:127-138.

Roozendaal B, Hernandez A, Cabrera SM, Hagewoud R, Malvaez M, Stefanko DP, Haettig J, Wood MA (2010) Membrane-associated glucocorticoid activity is necessary for modulation of long-term memory via chromatin modification. J Neurosci 30:5037-5046.

Saavedra A, Giralt A, Rué L, Xifró X, Xu J, Ortega Z, Lucas JJ, Lombroso PJ, Alberch J, Pérez-Navarro E (2011) Striatal-enriched protein tyrosine phosphatase expression and activity in Huntington's disease: a STEP in the resistance to excitotoxicity. J Neurosci 31:8150-8162.

Sala M, Perez J, Soloff P, Ucelli di Nemi S, Caverzasi E, Soares JC, Brambilla P (2004) Stress and hippocampal abnormalities in psychiatric disorders. Eur Neuropsychopharmacol 14:393-405.

Sapolsky RM (2000) The possibility of neurotoxicity in the hippocampus in major depression: a primer on neuron death. Biol Psychiatry 48:755-765.

Schrader LA, Birnbaum SG, Nadin BM, Ren Y, Bui D, Anderson AE, Sweatt JD (2006) ERK/MAPK regulates the $\mathrm{K}_{\mathrm{V}} 4.2$ potassium channel by direct phosphorylation of the pore-forming subunit. Am J Physiol Cell Physiol 290:C852-C861.

Sheline YI (2003) Neuroimaging studies of mood disorder effects on the brain. Biol Psychiatry 54:338-352.

Snyder EM, Nong Y, Almeida CG, Paul S, Moran T, Choi EY, Nairn AC, Salter MW, Lombroso PJ, Gouras GK, Greengard P (2005) Regulation of NMDA receptor trafficking by amyloid- $\beta$. Nat Neurosci 8:1051-1058.

Sweatt JD (2004) Mitogen-activated protein kinases in synaptic plasticity and memory. Curr Opin Neurobiol 14:311-317.

Tashev R, Moura PJ, Venkitaramani DV, Prosperetti C, Centonze D, Paul S, Lombroso PJ (2009) A substrate trapping mutant form of striatalenriched protein tyrosine phosphatase prevents amphetamine-induced stereotypies and long-term potentiation in the striatum. Biol Psychiatry 65:637-645.

Valjent E, Pascoli V, Svenningsson P, Paul S, Enslen H, Corvol JC, Stipanovich A, Caboche J, Lombroso PJ, Nairn AC, Greengard P, Hervé D, Girault JA (2005) Regulation of a protein phosphatase cascade allows convergent dopamine and glutamate signals to activate ERK in the striatum. Proc Natl Acad Sci U S A 102:491-496.

Wang Z, Neylan TC, Mueller SG, Lenoci M, Truran D, Marmar CR, Weiner MW, Schuff N (2010) Magnetic resonance imaging of hippocampal subfields in posttraumatic stress disorder. Arch Gen Psychiatry 67:296-303.

Yang CH, Huang CC, Hsu KS (2004) Behavioral stress modifies hippocampal synaptic plasticity through corticosterone-induced sustained extracellular signal-regulated kinase/mitogen-activated protein kinase activation. J Neurosci 24:11029-11034.

Yang CH, Huang CC, Hsu KS (2006) Novelty exploration elicits a reversal of acute stress-induced modulation of hippocampal synaptic plasticity in the rat. J Physiol 577:601-615.

Yehuda R, LeDoux J (2007) Response variation following trauma: a translational neuroscience approach to understanding PTSD. Neuron $56: 19-32$.

Yehuda R, Flory JD, Southwick S, Charney DS (2006) Developing an agenda for translational studies of resilience and vulnerability following trauma exposure. Ann N Y Acad Sci 1071:379-396.

Zhang Y, Venkitaramani DV, Gladding CM, Zhang Y, Kurup P, Molnar E, Collingridge GL, Lombroso PJ (2008) The tyrosine phosphatase STEP mediates AMPA receptor endocytosis after metabotropic glutamate receptor stimulation. J Neurosci 28:10561-10566.

Zhang Y, Kurup P, Xu J, Carty N, Fernandez SM, Nygaard HB, Pittenger C, Greengard P, Strittmatter SM, Nairn AC, Lombroso PJ (2010) Genetic reduction of striatal-enriched tyrosine phosphatase (STEP) reverses cognitive and cellular deficits in an Alzheimer's disease mouse model. Proc Natl Acad Sci U S A 107:19014-19019.

Zhu JJ, Qin Y, Zhao M, Van Aelst L, Malinow R (2002) Ras and Rap control AMPA receptor trafficking during synaptic plasticity. Cell 110:443-455. 